
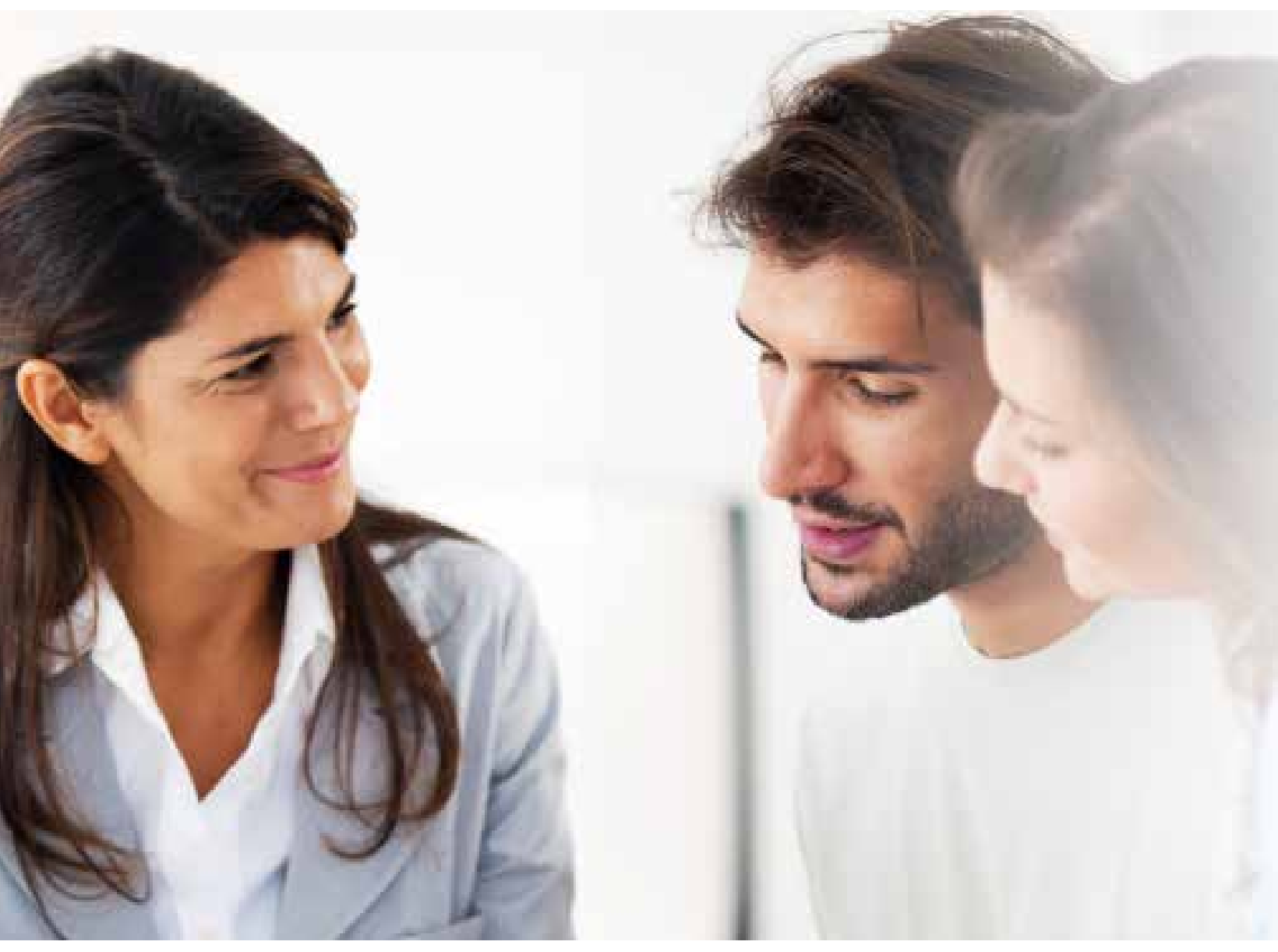

\title{
2014
}

\section{ABSTRACTS AND POSTERS}

\section{Canadian Pharmacists Conference Saskatoon, Saskatchewan}




\section{Table of Contents}

MOVING THE PROFESSION FORWARD ON SHIFTING SANDS: PRACTICE RESEARCH TO INFLUENCE POLICY

S1 Ontario Pharmaceutical Opinion Program: Initial experience

S2 PhINDMORE: Pharmacists' Innovative Drug Therapy Management Outcomes, Resource use and Economics

S3 Public attitudes towards the expanded scope of pharmacy practice in Nova Scotia

S4 SafetyNET-Rx Phase II: Analysis of medication incidents in community pharmacy

\section{ACTIVELY ENGAGING PHARMACISTS IN MEDICATION MANAGEMENT TO IMPROVE PATIENT OUTCOMES}

S5 Understanding how Albertan community pharmacists assess the appropriateness of medication therapy: A mixed methods approach

S6 Pharmacists' prescribing practices in Alberta: Report of a provincial survey

S7 Initial steps in developing benzodiazepine deprescribing guideline

S8 Experiences of Saskatchewan pharmacists and pharmacies providing professional services to long-term care homes

FOUNDATIONAL SYSTEM-WIDE RESEARCH: ONTARIO PHARMACY RESEARCH COLLABORATION (OPEN)

S9 Deprescribing in a family health team: A pilot study of chronic proton pump inhibitor (PPI) use

S10 Pharmacy students as immunizers: Review and policy recommendations

S11 Capturing the perspectives of policy makers, physicians and pharmacists about the evaluation of pharmacy services

S12 Developing and implementing more inclusive demographic survey questions

\section{LEVERAGING INNOVATION: MAXIMIZING THE ROLE OF PHARMACISTS IN IMMUNIZATIONS}

S13 Community pharmacist-administered influenza immunization improves patient access to vaccination 


\section{CANADIAN PHARMACISTS CONFERENCE \\ MAY 31 - JUNE 3 - SASKATOON, SK \\ ABSTRACTS AND POSTERS 2014}

S14 Relationship between personality and organizational culture in implementing advanced practice opportunities: Immunization as a case study

S15 Pharmacy students as immunizers: Review and policy recommendations

S16 Patient perspectives on pharmacists as immunizers: A scoping review

\section{LOWERING THE PRESSURE: PHARMACISTS' ROLE IN CARDIOVASCULAR INTERVENTIONS}

S17 Impact of community pharmacist interventions in hypertension management on patient outcomes: A randomized controlled trial

S18 A randomized trial of the effect of pharmacist prescribing on improving blood pressure in the community: The Alberta clinical trial in optimizing hypertension (RXACTION)

S19 Uptake of the Ontario Pharmacy Smoking Cessation Program, 2011-2013

S20 A community-based approach to dyslipidemia management: Pharmacist prescribing to achieve cholesterol targets (RxACT Study)

\section{POSTER ABSTRACTS}

S21 Pharmacists' perceptions of prescribing in two Canadian jurisdictions

S22 Developing, implementing and evaluating deprescribing guidelines for the elderly as a mixed methods approach

S23 Completeness of medication information in the outpatient referral process: A case for pharmacist involvement

S24 Impact of an online program to improve pharmacists' skills and confidence in providing patient care

S25 2006-2013 trends in adherence to antidepressants, cardiovascular disease medications and diabetes medications in a community pharmacy

S26 A community pharmacy-based point of care lipid screening program to improve cardiovascular disease risk management

S27 Chronic pain stakeholder engagement workshop

S28 ClereMed: A rapid prototyping project to build a mobile screening tool to identify adults 55 and over who have difficulty reading medication labels

S29 Community pharmacists' attitudes toward dispensing errors at community pharmacy settings in Central Saudi Arabia 
S30 Consumers' evaluation of community pharmacy services in Al-Ahsaa province, eastern region of Saudi Arabia

S31 Continuous quality assurance pilot project in Saskatchewan community pharmacies

S32 Drug shortages and patient safety

S33 Effectiveness of extracurricular journal clubs on pharmacy students' learning of evidencebased medicine and critical appraisal

S34 Handbook for a pilot study to reduce potential hospitalizations due to preventable drug-drug interactions

S35 Methadone medication incidents in community pharmacy

S36 Multi-incident analysis of medication incidents involving contraceptives

S37 The role of online/smartphone applications in Type II Diabetes Management: A qualitative study

S38 Controversy vaccination

S39 To crush or not to crush

S40 Developing a Recognition of Prior Learning (RPL) process for implementation in a PharmD bridging program

S41 Development of an interdisciplinary student managed, primary health care clinic in Scotland: A Canadian collaboration

S42 Pharmacist prescribing education: An international knowledge exchange study

S43 Pay-for-performance remuneration for pharmacist prescribers' management of hypertension: A pre-specified sub-study of the Alberta Clinical Trial in Optimizing Hypertension (RxACTION)

S44 Paying pharmacists for patient care: A systematic review of remunerated pharmacy clinical care services

S45 The validity of home blood pressure monitor validation studies

S46 The design of the Alberta Vascular Risk Reduction Community Pharmacy Project: RxEACH

S47 Economic analysis of the RxING study

S48 Pharmacist comfort with reporting quality-related events (QREs) of varying severity

S49 Preliminary findings of a study of experience with prescriptive authority (PA) services among Saskatchewan community pharmacists

S50 Preventing misunderstanding of medication labels: Establishment of a multilingual medication labeling service that can be provided by all staff, with the use of existing resources

S51 The clinical pharmacist as a team member within a Pulmonary Rehabilitation Program (PRP) 
S52 The validity of information-sharing about pharmacists' education, skills and scope of practice in informing public perceptions

S53 Public attitudes of community pharmacy reward points and incentive programs

S54 Understanding pharmacists' personality traits using the Big Five Inventory

S55 Understanding pharmacy culture using the Organizational Culture Profile

S56 Pharmacists' working conditions in British Columbia

S57 Systematic review of pharmacist role and interventions in long-term care facilities

S58 Inclusion of information regarding older adults in drug product monographs

S59 Measuring the value of community pharmacists' provision of professional services: A methodology using pharmacy billing data

S60 Pharmacists' perceptions of the impact of Computerized Prescriber Order Entry (CPOE) on their practice 


\section{Ontario Pharmaceutical Opinion Program : Initial experience}

Bryan Gray, BScPharm, RPh

OBJECTIVES: $\quad$ Assess whether the Ontario Pharmaceutical Opinion Program is both feasible from a practical and financial perspective.

METHODS: As a third-year pharmacy student, I conducted medication reviews and provided recommendations between June and August 2012 in a community pharmacy in Thunder Bay. Patients who met the eligibility criteria and might have benefited from a medication review were offered a review at that time or the opportunity to schedule an appointment for a later date. Recommendations were sent to the patient's primary prescriber.

RESULTS: $\quad$ In 3 months, 87 pharmaceutical opinions were provided for 39 patients (21 male) and faxed to 26 different prescribes after completing 522 medication reviews. From all the recommendations provided, 50 received a response from prescribers and 38 were eligible for reimbursement. This generated $\$ 570$ in revenue from eligible responses. Unfortunately, $\$ 735$ was no remunerated due to lack of prescriber response or ineligibility. The majority of pharmaceutical opinions were for cardiovascular disease and diabetes. A cost analysis demonstrated that for every $\$ 1.00$ spent on costs (ex. pharmacist wage), \$2.61 was generated.

CONCLUSIONS: This study demonstrated that the pharmaceutical opinion program is practically and financially feasible. Developing strong relationships with prescribers will be critical as the scope of practice for pharmacists continues to expand. As minor ailments prescribing, adaption and substitutions become more common; prescribers must be able to trust the pharmacist to make evidence-based, patient-centered decisions. Even when pharmacists are able to access the patient's medical record, inter-professional collaboration will still be essential. The pharmaceutical opinion program is a great first step for the profession, but more must be taken to reach our full potential. 


\section{PhINDMORE: Pharmacists' Innovative Drug Therapy Management Outcomes, Resource use and Economics}

Nicole Tsao, BScPharm; MScPharm; Kathy Li, PhD; John Shaske, BScPharm; Larry Lynd, BSP, PhD; Carlo Marra, PharmD, PhD

OBJECTIVES: As part of a pilot project, medication management (MM) services in $B C$ are being reimbursed at one pharmacy. We hypothesize that interventions during MM will improve health and result in reduced health care utilization. To characterize the outcomes of MM services performed by pharmacists at a single, innovative pharmacy and determine the impact on health care utilization.

METHODS: Descriptive statistics on patient demographics, medical conditions and medications were performed on a cohort of patients receiving MM from January 2012 to October 2013 and actions taken as a result of MM sessions were evaluated. Health resource utilization saved or incurred as a result of MM was also estimated.

RESULTS: $\quad$ The cohort consisted of 2085 patients, 58\% female and the mean age was 60 years. Mean number of MM sessions was 2.3 (SD 2.1) per patient; patients $\geq 65$ years received more (mean 2.9 vs. 1.9, $\mathrm{p}<0.001$ ). The top 5 conditions were hypertension (8.8\%), anxiety (4.5\%), pain (4.3\%), asthma (3.9\%) and depression (3.8\%). The top 5 actions taken were to provide chronic disease (70\%) and/or drug or device education (47\%), to initiate an OTC medication (16\%) and to change the quantity (11\%) and/or dose of a medication (10\%). There were also 105 referrals to physicians and 1 referral to the ED; MM was estimated to result in avoidance of 1033 physician visits, 11 ED visits and 9 hospitalizations.

CONCLUSIONS: Pharmacists provided MM services to patients with mostly chronic conditions. These services appeared to result in avoidance of health care utilization. 


\section{Public attitudes towards the expanded scope of pharmacy practice in Nova Scotia}

Todd Boyle, PhD; Thomas Mahaffey, PhD; Kellie Duggan, BSc; Bev Zwicker, BSc(Pharm); Andrea Bishop, PhD; Andrea Murphy, PharmD; James Barker, PhD; Bobbi Morrison, PhD; Neil Mackinnon, PhD; Megan MacDonald, BBA Student

OBJECTIVES: $\quad$ Pharmacists expanded scope of practice (ESOP) has the potential to increase timely access to important health care services. This research explores public attitudes towards ESOP in Nova Scotia.

METHODS: $\quad$ ESOP attitudes were captured using an in-pharmacy intercept survey and an online survey. The survey focused on four key ESOP elements/services: prescribing for minor ailments; medication reviews; injections and vaccinations; and prescription renewals. Open-ended and 5 -point scale ( 1 = strongly disagree; 5 = strongly agree) questions were used to obtain perceptions of: pharmacists' ability to deliver ESOP; comfort level with pharmacists delivering such services; and the likelihood of using such services. Thematic analyses, descriptive statistics and comparisons based on practice awareness (used, aware, not aware) using MANOVA were performed.

RESULTS: $\quad$ A total of 385 surveys were obtained (online $n=237$; in-pharmacy $n=148$ ). Pharmacist knowledge/skills (mean $=4.62$ ) and medication history on file (mean $=4.52$ ) greatly impacted the public's decision to use expanded services, whereas pharmacist gender $($ mean $=2.14)$ and age $($ mean $=2.39)$ impacted the least. The public reported high levels of comfort with pharmacists performing each service and believe that pharmacists have the knowledge to properly deliver such services. However, a number of statistically significant differences in perceptions were found based on awareness of each specific service.

CONCLUSIONS: This research provides a benchmark of attitudes related to ESOP. Our findings indicate that the public is very interested in ESOP, believe they will benefit and that pharmacists have the necessary knowledge and skills. Attitudes, however, may differ based on awareness of specific expanded services. 


\section{SafetyNET-Rx Phase II: Analysis of medication incidents in community pharmacy}

Certina Ho, RPh, BScPhm, M.I.St., M.Ed.; Jim Kong, BSc, PharmD Candidate; Roger Cheng RPh, BScPhm, PharmD; Todd Boyle, PhD; Thomas Mahaffey, PhD; Neil MacKinnon, BSc (Pharm), MSc (Pharm), PhD, FCSHP

OBJECTIVES: $\quad$ SafetyNET-Rx is a continuous quality improvement (CQI) program for community pharmacies in Nova Scotia. Its main objective is to pinpoint the underlying systembased vulnerabilities that lead to near misses or medication incidents and to facilitate open discussion on appropriate intervention strategies for enhancing medication safety.

METHODS: $\quad$ From March 2010 to April 2013, 26,744 medication incidents were voluntarily reported by 84 community pharmacies participating in Phase II of the SafetyNET-Rx pilot project. Quantitative analysis was conducted on all 26,744 incidents, while qualitative, multi-incident analysis was conducted on a subset of 132 incidents.

RESULTS: $\quad$ Of the 26,744 incidents reported, $88.6 \%(23,864$ of 26,744$)$ were near misses, $10.9 \%$ ( 2928 of 26,744 ) caused no patient harm and $0.486 \%$ (132 of 26,744$)$ resulted in patient harm. Of these 132 incidents, only $0.007 \%$ (2 of 132) of incidents resulted in severe patient harm that health care professional intervention was required. The prescription order-entry stage and the dispensing stage were the two most commonly reported stages where medication incidents occurred. The top five reported medications were acetaminophen with codeine, rosuvastatin, salbutamol, fluticasone and amoxicillin. Multi-incident analysis found numerous potential contributing factors to medication incidents, including look-alike/sound-alike drug names, alert fatigue, dangerous abbreviations and illegible prescriptions.

CONCLUSIONS: SafetyNET-Rx was a CQI initiative with an intention to investigate the factors that can contribute to medication incidents in community pharmacy settings. The goal is to improve patient safety through the analysis of incidents and to disseminate results and lessons learned to health care practitioners for future prevention of near misses or errors. 


\section{Understanding how Albertan community pharmacists assess the appropriateness of medication therapy: A mixed methods approach}

Mohammad Nasir, BSc Pharm, MSc, Clinical Pharmacy; Lisa Guirguis, BSc Pharm, MSc, PhD

OBJECTIVES:

METHODS:

RESULTS:

CONCLUSIONS: This study suggests that pharmacists focus on technical issues particularly for refills and may miss opportunities for patient care beyond medication safety. Pharmacists make almost a complete assessment for new prescriptions and less effort is made for chronic refills. This pilot study highlights the need for further study. 


\section{Pharmacists' prescribing practices in Alberta: Report of a provincial survey}

Christine Hughes, BScPharm, PharmD, FCSHP; Mark Makowsky, BSP, PharmD; Cheryl Sadowski, BSc(Pharm), PharmD; Theresa Schindel, BSP, MCE, FCSHP; Nese Yuksel, BScPharm, PharmD, FCSHP, NCMP

OBJECTIVES: $\quad$ Alberta has the broadest model prescribing in Canada. The objective was to characterize pharmacists prescribing practices in Alberta.

METHODS: $\quad$ This cross-sectional web-based survey was conducted in a random sample of pharmacists on the Alberta College of Pharmacists clinical register. The survey was developed in four stages with evidence for reliability and validity. A pre-notification letter, small incentive, five reminders and telephone follow-up were employed. The survey was available from April 19 to June 10, 2013. Analysis was descriptive. Prescribing behaviour and beliefs were compared between practices using ANOVA and chi-square.

RESULTS: $\quad$ A total of 350 pharmacists completed the survey (51\% response rate; 692 eligible) from various settings including community (76.9\%), hospital (11.1\%) and consultant practice (i.e., primary care team or long term care) (12.0\%). Twenty-two pharmacists (6.3\%) had additional prescribing authorization (APA). Twenty-three pharmacists (6.6\%) did not prescribe as they practiced in a team or consulting role, or preferred to fax prescription requests to physicians. The most common forms of prescribing were ensuring continuity of therapy (92.3\%), adapting (73.4\%) and medication substitution due to shortage (80.5\%). Pharmacists with APA were more likely to prescribe to adjust ongoing medications than initiate a new medication. Community pharmacists were more likely to prescribe to renew medications. Hospital and consultant pharmacists were more likely to adapt prescriptions, use APA and view prescribing as an expanded professional role.

CONCLUSIONS: Pharmacists have embraced prescribing in a form that mirrors their practice environment. Future research should evaluate the impact of prescribing from a patient, physician and health system perspective. 


\section{Initial steps in developing benzodiazepine deprescribing guideline}

Cheryl Sadowski, BSc (Pharm), PharmD, FCSHP; Barbara Farrell, BScPhm, PharmD, FCSHP; Anne Holbrok, MD, PharmD, MSc, FRCP(C); Carlos Rojas-Fernandez, BSc(Pharm), PharmD; Natalie Ward, PhD (ABD); Corey Tsang, BSc, BScPhm candidate; Lisa Pizzola, BSc, MSc

OBJECTIVES: The purpose of this presentation is to describe the process used to initiate development and implementation of an interprovincial benzodiazepine deprescribing guideline.

METHODS: $\quad$ Canadian Institute of Health Research funding was received to conduct an expert and stakeholder 1.5 day planning meeting regarding interprofessional approaches to benzodiazepine tapering. Objectives were to discuss challenges related to benzodiazepines, critique a proposed interprofessional model for deprescribing benzodiazepines and identify interventions and evaluation strategies for implementing the model. Discussions and presentations were recorded and transcribed. Activities and outcomes are presented descriptively.

RESULTS: $\quad$ Nineteen local service providers and 14 stakeholders, in addition to the 5 members of the research team, participated in the meeting. Key messages identified were: the need for an adaptable model, challenges of implementing changes in a structured and highly regulated long-term care environment, consideration of deprescribing on the health care provider and the health system, the quality of evidence for benzodiazepine tapering and finally, the challenge of driving behavior change in practice. Development and implementation of a benzodiazepine deprescribing guideline was discussed. The RE-AIM (reach, effectiveness, adoption, implementation, maintenance) model (a 5 dimensional framework that increases chances of an intervention being successful in real world settings) was chosen to frame further intervention and evaluation work.

CONCLUSIONS: There is support and receptivity to guidelines for benzodiazepine deprescribing. Evidence to support benzodiazepine tapering is available to guide development of a deprescribing guideline. The primary concern is related to usability of such a guideline and implementing the practice into a culture of prescribing. 


\section{Experiences of Saskatchewan pharmacists and pharmacies providing professional services to long-term care homes}

Jason Perepelkin, PhD; Julie Bareham, BSP

OBJECTIVES:

In community pharmacy practice a subset of pharmacists and pharmacies provide services to long-term care and special care (LTC) homes. Offering distribution and services to LTC homes, impacts practice in ways that are unique from regular practice. The objectives of this study were to identify how community pharmacists were performing medication reviews and to identify any barriers or challenges to performing medication reviews.

METHODS: $\quad$ During January and February 2014 data was collected via an online, self-administered questionnaire. A fax and follow up e-mail, that included an overview of the study and a link to the questionnaire, was sent to community pharmacies in Saskatchewan, with endorsements from the Pharmacists Association of Saskatchewan and the Saskatchewan College of Pharmacists. Questions focused on four main areas: workflow; economic model; pharmacist skills; and interdisciplinary/team approach.

RESULTS: $\quad$ One-third (31\%) of respondents reported serving LTC homes with $>200$ patients, while another third (29\%) serve 51-100 patients. Most (77\%) respondents reported having a dedicated pharmacist to perform LTC resident medication reviews. Respondents reported the biggest challenge/limitation of performing LTC resident medication reviews was the lack of appropriate reimbursement for pharmacist time (66\%). One-third (32\%) of respondents reported completing LTC medication reviews in the pharmacy, followed by other (29\%) that included partially at the pharmacy, partially at the LTC home. Three-quarters of respondents (76\%) reported having a dedicated pharmacist to attend resident care conferences at LTC homes.

CONCLUSIONS: This study provides an overview of pharmacies providing LTC services that will help inform policy. With changes in requirements for medication reviews and contracts for LTC homes, supports are needed to ensure pharmacies are providing optimal care while being adequately remunerated for services provided. 


\section{Deprescribing in a family health team: A pilot study of chronic proton pump inhibitor (PPI) use}

Kate Walsh, BScH, BScPhm, RPh; Debbie Kwan, BScPhm, MSc; Patricia Marr, BScPhm, PharmD;

Christine Papoushek, PharmD; Dr. Kirk Lyon, MD

OBJECTIVES: $\quad$ Studies show PPIs are often used inappropriately (i.e. without an indication or for longer durations than recommended), however, few tools exist to guide their reassessment and deprescribing. This study aimed to develop, implement and evaluate a PPI deprescribing process and tool in an academic family medicine clinic.

METHODS: $\quad$ The primary care providers (PCPS) of adult patients taking a PPI for $\geq 8$ weeks with an upcoming periodic health exam (PHE) were sent a reminder message to reassess therapy via the electronic medical record. A PPI Deprescribing Tool was also uploaded into the patient's chart to serve as a second reminder and to guide reassessment and deprescribing (where indicated). Follow up data collection occurred 10 weeks after the PHE to determine changes to PPI use. A PCP survey assessed the usability and barriers to implementing the intervention.

RESULTS: $\quad$ The majority of the 46 included patients were on standard dose PPI for GERD for > 1 year. Ninety-three percent had their PPI reassessed during the PHE which resulted in 63\% having their PPI stopped or stepped down to an alternate therapy. Survey respondents found the reminder message more useful than the PPI Deprescribing Tool. The "Tool", however, was frequently cited as a facilitator to adopting the process in their practice.

CONCLUSIONS: PCP utilization of the process, "Tool" and user feedback were positive. Future research should validate this intervention on a larger scale. This project adds to the deprescribing literature by demonstrating that guidance tools may assist in adoption of the practice. 


\section{Pharmacy students as immunizers: Review and policy recommendations}

Sarah Johnson, BSc, BScPhm candidate; Nancy Waite, BScPhm, PharmD, FCCP; Eric Schneider, BScPharm, PharmD, BCPS

OBJECTIVES: Despite universal influenza vaccine availability, vaccination rates have stagnated at suboptimal levels. To address this issue, several provinces and all US states authorize pharmacists to provide influenza vaccination. Pharmacy students are another potential vaccine administrator and inclusion of this opportunity during their undergraduate training has the potential to both assist pharmacist vaccination efforts and improve students' readiness to provide these services upon graduation. The goal of this initiative was to summarize pharmacy students-as-immunizers literature and regulatory authority and make Canadian policy recommendations with the available evidence.

METHODS: Data was collected regarding which Canadian provinces and US states have legislation allowing pharmacy students to immunize, which vaccines are they permitted to give, what training is required and what level of supervision is mandated. A literature review was also conducted to examine the student, patient and community benefits of pharmacy students-as-immunizers.

RESULTS: $\quad$ Four Canadian provinces and 30 US states have legislation permitting students to administer vaccines. In all cases, students must have completed immunization training and be under the supervision of a pharmacist authorized to immunize. The literature provides a range of examples of strategies for students-as-immunizers including embedding in rotations, student-run clinics and involvement in health fairs and community based clinics. Students on campus, general public and high risk groups were targeted populations.

CONCLUSIONS: Students-as-immunizers is a mechanism that should be considered to promote the role of the pharmacist and increase the availability and accessibility of vaccines. Specific policy recommendations will be summarized. 


\title{
Capturing the perspectives of policy makers, physicians and pharmacists about the evaluation of pharmacy services
}

\author{
Lisa Dolovich, BScPhm, PharmD, MSc; Karen Slonim, PhD; Linda MacKeigan, BScPhm, PhD; Tejal Patel, \\ PharmD; Nancy Waite, BScPhm, PharmD, FCCP; Feng Chang, BScPhm, PharmD; Barb Farrell, PharmD
}

OBJECTIVES: $\quad$ To capture expert perspectives on the critical structure, indicators and processes of a pharmacy services evaluation framework and to gain a better understanding of the barriers and facilitators to the development and uptake of this framework.

METHODS: Descriptive qualitative study with purposefully selected stakeholders (academics, practitioners and policy makers) from across Canada participating in semi-structured interviews. Data were organized using an iteratively developed codebook within NVivo software. Data analysis focused on emerging themes related to an evaluation framework structure, indicators and outcomes as well as barriers and facilitators to its use.

RESULTS: $\quad$ Thirty-five key informants were identified (13 academics, 14 policy makers, 8 practitioners). Preliminary findings from twenty interviews are available. The majority of participants identified a dearth of policy-informative evidence on the impact of existing pharmacy services. Participant responses varied considerably with regard to appropriate measures for evaluating pharmacy services. However, emergent themes of capturing the patient experience, outcomes important to patients and the role of pharmacists as important and effective communicators were identified. Consensus was less apparent in areas of the preferred unit of analysis (pharmacist vs. pharmacy) and preferred types of outcome measures (disease-specific vs. quality indicators).

CONCLUSIONS: This project has highlighted the importance of gathering input from diverse stakeholders in advance of creating an evaluation framework. The process is expected to encourage consensus building and consolidation of priorities across stakeholder groups to improve evidence-informed policy making. 


\section{Developing and implementing more inclusive demographic survey questions}

Feng Chang, PharmD; Katie M. Cook, MA; Martin Cooke, PhD; Lisa McCarthy, PharmD; Beth Sproule, PharmD; Nancy Waite, PharmD

OBJECTIVES: Vulnerable populations are more likely to incur negative health outcomes and this should be considered in pharmacy practice research. To ensure that survey demographics were collected in a consistent manner that was inclusive of vulnerable populations, Ontario Pharmacist Research Collaboration (OPEN)'s Gender and Vulnerable Populations (GVP) team developed best practice survey questions that were provided to researchers via several venues.

METHODS: The GVP team (whose expertise includes pharmacy, demography, sociology and community psychology) identified demographic areas that represented vulnerable populations. Team members proposed best practice questions within their area of expertise. The team then provided a summary of questions and rationale. OPEN researchers provided input regarding preferred mechanisms for dissemination and educational tools were developed based on this feedback.

RESULTS: $\quad$ The GVP team identified best practice questions in the areas of sex/gender, sexual orientation, age, geographic location, race and ethnicity, Aboriginal identity and socioeconomic factors including education and income. Dissemination included 1) sending the recommended questions to researchers via email, 2) posting an online learning module that included a) suggested survey questions, b) a background document including literature and other potential questions and c) an asynchronous presentation on sex/gender and intersectionality, an area identified as less familiar to OPEN researchers and 3 ) offering to work with any research teams during survey development and analysis.

CONCLUSIONS: To ensure that vulnerable populations were considered in survey research design, OPEN, a large multi-institutional pharmacy practice research network, developed recommended survey questions and an educational process to inform OPEN researchers and their research. 


\section{Community pharmacist-administered influenza immunization improves patient access to vaccination}

John Papastergiou, BScPhm; Chris Folkins, BScPhm, PhD; Wilson Li, BScPhm, CDE; John Zervas, PharmD

OBJECTIVES: To describe the demographic characteristics and risk factors of patients receiving influenza vaccination in community pharmacies and to understand patient experiences and perceptions surrounding being vaccinated by a pharmacist.

METHODS: $\quad$ Survey data was collected by research pharmacists at 4 different retail pharmacy locations in Toronto over a period of 8 weeks during October and November 2013. Participation in the survey was voluntary and all patients vaccinated by research pharmacists were invited to complete a survey following immunization.

RESULTS: $\quad$ Over the course of the study, 2498 vaccine doses were administered across all study sites and 1502 surveys were completed. Our data showed a high degree of patient satisfaction, with $91.8 \%$ of patients indicating they were "very satisfied" with the pharmacist's injection technique and the services they received. Furthermore, 85.7\% of patients were "very comfortable" being vaccinated by a pharmacist and $98.7 \%$ of patients reported they would recommend that friends and family be vaccinated by a pharmacist. Convenience and accessibility were major determinants of patient satisfaction, as evidenced by $45.7 \%$ of all written comments specifically addressing these factors. Of the patients surveyed, $24.7 \%$ were not regular annual vaccine recipients and $46.8 \%$ were classified as high risk for influenza complications according to Public Health Agency of Canada criteria. Notably, $27.5 \%$ of total patients and $21.1 \%$ of high risk patients reported that they would not have been immunized this year if pharmacybased vaccination were not available.

CONCLUSIONS: Our findings suggest that pharmacists provide a highly convenient and accessible option for seasonal flu vaccination that is viewed favorably by patients. Administration of the flu vaccine by pharmacists has the potential to positively impact public health by improving vaccination rates amongst high-risk patients, first time or occasional vaccine recipients and patients who may not otherwise have an opportunity to be vaccinated. 


\section{Relationship between personality and organizational culture in implementing advanced practice opportunities: Immunization as a case study}

Meagen Rosenthal, MA; Carlo Marra, PharmD, PhD; Bob Nakagawa, Registrar of the College of Pharmacists of British Columbia; Nicole Tsao, MPharm; Larry Lynd, PhD; Ross Tsuyuki, PharmD

OBJECTIVES: To determine the association between personality and organizational culture and frequency of immunization.

METHODS: $\quad$ An online survey was distributed to all pharmacists registered in BC. The survey was composed of three sections: 1) demographics including frequency of immunization per month,2) Organizational Culture Profile(OCP), which includes scores for competitiveness, social responsibility, supportiveness, innovation, rewards, performance and stability; 3) Big Five Inventory (BFI) which includes 5 point scores for extraversion, agreeableness, conscientiousness, openness and neuroticism. Ordinal logistic regression was used to determine the association between frequency of immunization and scores from the BFI and OCP.

RESULTS: $\quad$ A total of 945 pharmacists completed all surveys; $61 \%$ were female and mean age was 42 years (SD 12). The distribution of the frequency of immunization in the previous month was $45 \%$ for never, 38\% for 1 to 10 times, $17 \%$ for greater than 10 times. In the gender adjusted regression model, the frequency of immunization was associated with the personality traits extraversion (OR $1.3595 \% \mathrm{Cl} 1.11$ to 1.64 ) and conscientiousness (OR $0.78,95 \% \mathrm{Cl} 0.60$ to 0.99 ) and the cultural factors of workplace competitiveness (OR $1.6795 \% \mathrm{Cl} 1.40$ to 1.98 ) and workplace stability (OR 0.66, 95\% Cl 0.53 to 0.82). Interactions were not significant.

CONCLUSIONS: Pharmacists who scored highly on extraversion or felt that pharmacy culture is competitive in nature provided more immunizations, than those who scored highly on conscientiousness or felt that the culture was stable. These results suggest the frequency of immunizations is associated with both personality traits and workplace pharmacy culture. 


\section{Pharmacy students as immunizers: Review and policy recommendations}

Sarah Johnson, BSc, BScPhm candidate; Nancy Waite, BScPhm, PharmD, FCCP; Eric Schneider, BScPharm, PharmD, BCPS

OBJECTIVES: Despite universal influenza vaccine availability, vaccination rates have stagnated at suboptimal levels. To address this issue, several provinces and all US states authorize pharmacists to provide influenza vaccination. Pharmacy students are another potential vaccine administrator and inclusion of this opportunity during their undergraduate training has the potential to both assist pharmacist vaccination efforts and improve students' readiness to provide these services upon graduation. The goal of this initiative was to summarize pharmacy students as immunizers literature and regulatory authority and make Canadian policy recommendations with the available evidence.

METHODS: $\quad$ Data was collected regarding which Canadian provinces and US states have legislation allowing pharmacy students to immunize, which vaccines are they permitted to give, what training is required and what level of supervision is mandated. A literature review was also conducted to examine the student, patient and community benefits of pharmacy students as immunizers.

RESULTS: $\quad$ Four Canadian provinces and 30 US states have legislation permitting students to administer vaccines. In all cases, students must have completed immunization training and be under the supervision of a pharmacist authorized to immunize. The literature provides a range of examples of strategies for students as immunizers including embedding in rotations, student-run clinics and involvement in health fairs and community based clinics. Students on campus, general public and high risk groups were targeted populations.

CONCLUSIONS: Students-as-immunizers is a mechanism that should be considered to promote the role of the pharmacist and increase the availability and accessibility of vaccines. Specific policy recommendations will be summarized. 


\section{Patient perspectives on pharmacists as immunizers: A scoping review}

Olivia Sergeant, BScPhm candidate; Lisa Wenger, BA, MA, PhD; Eric Schneider, BScPharm, PharmD, BCPS; Nancy M. Waite, BScPhm, PharmD

OBJECTIVES: $\quad$ Although an important Public Health strategy, the provision of flu shots by pharmacists in Canada is still in its infancy. To ensure safe, effective and appropriate steps forward, it is important to understand public opinion of this service. To that end, a scoping review was completed to provide an overview of the literature on patient perspectives, attitudes and knowledge regarding pharmacists as immunizers.

METHODS: $\quad$ A scoping review of published and grey literature was completed using Arksey and O'Malley's (2005) methodological framework. Collected data was grouped based on common themes allowing for the identification of knowledge gaps and the development of suggestions/recommendations for future research and possible next steps regarding the understanding of patient outlooks on pharmacists as immunizers.

RESULTS: $\quad$ Research has predominantly used quantitative methods to examine (a) levels of knowledge/awareness regarding pharmacists as immunizers (b) experiences of those with a previous experience of pharmacist-administered immunization and (c) barriers/enablers to use of pharmacists as immunizers. This literature indicates that while awareness levels are low among the general population, satisfaction is high among those immunized by a pharmacist and patients appreciate the accessibility and convenience of pharmacy-based immunizations. Beyond the need for promoting pharmacist immunization services, barriers to be addressed include patient concerns regarding privacy and concerns about pharmacist qualifications.

CONCLUSIONS: Although there is detailed information on patient experiences post-vaccination, future research could examine the perceptions of those that have not accessed services (general population) and pursue in-depth, qualitative understandings of patients' hesitance to utilize pharmacies for immunizations. 


\title{
Impact of community pharmacist interventions in hypertension management on patient outcomes: A randomized controlled trial
}

\author{
Nedzad Pojskic, HonBSc, MSc, PhD; Mark Jackson, BScPhm, RPh; Allan Malek, BSc Biol, BScPhm; Sal \\ Cimino, BScPhm, RPh
}

OBJECTIVES: The primary objective of this study was to examine the impact of Ontario pharmacists' interventions in hypertension management on patient health outcomes. Secondary objective was to examine the feasibility of implementing a chronic disease management program in a private sector drug plan.

METHODS: $\quad$ This study consisted of a 6-month, prospective, randomized controlled trial conducted in 38 community pharmacies (11 independent/banner; 27 chain/franchise) from four regions in Ontario. The pharmacies were randomly allocated to either of the two study groups in a 1:1 ratio. One hundred and fifty-three patients who were Green Shield Canada drug plan recipients and had uncontrolled hypertension were recruited for this study. They met with their pharmacist on a monthly basis for a total of 7 times during the study period. Intervention group pharmacists provided patients with a comprehensive disease management program for hypertension, including medication review and drug therapy optimization, lifestyle education (nutrition, physical activity, smoking, etc.) and maximizing adherence to medication therapy. Control group patients were provided with standard dispensing services and counseling on new and repeat prescriptions. Adherence and drug cost information was obtained through Green Shield Canada's prescription claims database; all other data was collected by study pharmacists.

RESULTS: $\quad$ One hundred and eighteen patients completed the study, majority (79) of who were in the intervention group. Intervention group patients had greater improvement than control patients from baseline to study endpoint for all outcome measures. Systolic blood pressure declined by $13.5 \mathrm{~mm} \mathrm{Hg}$ in the intervention group compared to $5 \mathrm{~mm} \mathrm{Hg}$ in the control group $(\mathrm{p}<0.001)$. The proportion of patients with overall blood pressure control increased by $56.5 \%$ in the intervention group, compared to only $17.5 \%$ in the control group $(p<0.001)$. Self-reported adherence to medication therapy increased by $15 \%$ in the intervention group compared to only $2.2 \%$ in the control group, a difference that approached statistical significance $(p=0.08)$. The overall cost of antihypertensive medication therapy declined by $31.2 \%$ in the intervention and only $5.2 \%$ in the control group ( $p=0.055$ ). Patients reported a uniformly high level of satisfaction with pharmacist services, particularly the lifestyle counseling component. Twenty four percent of the employed patients reported being more productive at work as a result participating in the study.

CONCLUSIONS: A community pharmacist-led hypertension management program improved patient blood pressure and adherence to medication therapy and substantially reduced the overall cost of antihypertensive drug therapy. This study has demonstrated that such a program can be feasibly implemented in a private sector drug plan. 


\section{A randomized trial of the effect of pharmacist prescribing on improving blood pressure in the community: The Alberta clinical trial in optimizing hypertension (RXACTION)}

Ross Tsuyuki, BScPharm, PharmD, MSc, FCSHP, FACC; Sherilyn Houle, BSP, PhD;

Theresa Charrois, BScPharm, ACPR, MSc; Michael Kolber, BSc, MD, CCFP, MSc; Finlay McAlister, MD, MSc; Meagen Rosenthal, BA, MA, PhD(c); Richard Lewanczuk, MD, PhD; Dale Cooney, BScPharm

OBJECTIVES: Hypertension control rates in Canada remain suboptimal. Pharmacist prescribing is a new option for improving hypertension care. To determine the impact of pharmacist care (including prescribing), on systolic blood pressure (SBP) in patients with uncontrolled hypertension.

METHODS: $\quad$ Design: Randomized controlled trial with patients as the unit of randomization, with care provided by 22 Alberta pharmacists Alberta with prescribing authorization. Patients: Adults with BP above recommended targets (>140/90 or $>130 / 80 \mathrm{mmHg}$ if diabetic) based upon measurements over multiple visits. Enhanced Care: Pharmacist assessment, BP wallet card, education on hypertension, pharmacist prescribing of antihypertensive drugs and laboratory monitoring using current Canadian guidelines and monthly follow-up visits dropping down to every 3 months if target BP was achieved for 2 consecutive visits. All patients were followed for 6 months. Control: Wallet card for BP recording, patient information materials on hypertension and no study specific followup. Outcomes: Primary outcome was the difference in change in systolic BP between the intervention and control groups at 6 months.

RESULTS: $\quad$ A total of 248 patients were randomized to the study. The mean (SD) patient age was 63.5 (12.7) years, 48.8\% were male and the mean (SD) systolic/diastolic BP was 149.7(13.6)/83.4(11.5) $\mathrm{mmHg}$ at baseline. The intervention group had an adjusted mean (SE) reduction in systolic BP at 6 months of 18.0 (1.4) $\mathrm{mmHg}$ compared with 11.0 (2.1) $\mathrm{mm} \mathrm{Hg}$ in the control group ( $\mathrm{p}=0.005)$.

CONCLUSIONS: Pharmacist prescribing for patients with uncontrolled hypertension resulted in a statistically and clinically significant reduction in systolic blood pressure when compared to usual care. 


\section{Uptake of the Ontario Pharmacy Smoking Cessation Program, 2011-2013}

Lindsay Wong, BScPhm/PharmD student; Andrea M. Burden, MA; Mina Tadrous, PharmD, MS; Nedzad Pojskic, PhD; Lisa Dolovich, PharmD, MSc; Andrew Calzavara, MSc; Yan Yun Liu, MSc; Suzanne M. Cadarette, $P h D$

OBJECTIVES: $\quad$ To describe the uptake of the Ontario Pharmacy Smoking Cessation Program since its launch in September 2011.

METHODS: $\quad$ We leveraged administrative claims data in Ontario to describe public drug plan beneficiaries receiving and pharmacies providing smoking cessation services, 2011/092013/10. Descriptive characteristics were summarized, stratified by drug plan group: seniors (aged 65+ years), or social assistance. Trends over time were examined by plotting the number of services, unique patients and unique pharmacies by month. We then examined use of follow-up services and prescription smoking cessation medication.

RESULTS: $\quad$ We identified 7767 residents receiving pharmacy smoking cessation services: $28 \%$ seniors (mean age $=70, \mathrm{SD}=4.8 ; 53 \%$ male) and $72 \%$ social assistance (mean age $=44$ years, $\mathrm{SD}=11.8 ; 48 \%$ male). Patient enrolment increased steadily over time with an average increase of 311 (SD=61) patients per month and one third $(n=1,253)$ of pharmacies participated. Regions with the highest proportion of participants were: Erie St. Clair (17\%) and Hamilton Niagara Haldimand Brant (17\%). Sixteen percent of all patients received another pharmacy service (e.g., MedsCheck) on the same day as smoking cessation baseline assessment. Among 4,167 patients with one year of data, $56 \%$ received follow-up services (60\% seniors, 55\% social assistance) and $74 \%$ received a prescription smoking cessation medication.

CONCLUSIONS: Program enrolment has increased steadily since its launch, yet only a third of pharmacies participated and only 56\% of patients enrolled received follow-up services. Ontario may consider strategies to improve uptake and use of follow-up services, such as public awareness campaigns and expanding access to include non-public drug plan beneficiaries. 


\section{A community-based approach to dyslipidemia management: Pharmacist prescribing to achieve cholesterol targets (RxACT Study)}

Meagen Rosenthal, MA; Glen Pearson, BSc, BScPhm, PharmD, FCSHP; Ross Tsuyuki, BSc(Pharm), PharmD, MSC, FCSHP, FACC

OBJECTIVES: Dyslipidemia is an important modifiable risk factor for cardiovascular disease. Despite strong evidence and clear practice guidelines, it remains sub-optimally treated. Pharmacists are frontline primary care professionals and, with expanded scopes of practice, could identify and treat patients with dyslipidemia. To evaluate the effect of pharmacist prescribing and follow-up in patients with dyslipidemia not at recommended treatment targets.

METHODS: Design: Randomized trial of pharmacist prescribing vs. usual care. Setting: Fourteen community pharmacies in Alberta. Population: Adults with uncontrolled dyslipidemia (treated or untreated) as defined by the 2009 Canadian Dyslipidemia Guidelines. Intervention: Pharmacists assessed patients' cardiovascular risk, reviewed LDL-c control and developed treatment goals, including prescribing lipid-lowering medications. Follow-up was at 6, 12, 18 and 24 weeks. Control: Patients received usual pharmacist and physician care, a copy of their lab results and a pamphlet on cardiovascular disease. Follow-up was at 12 and 24 weeks.

RESULTS: $\quad$ We enrolled 99 patients with a mean (SD) age of 63 years (13), 49\% male and mean baseline LDL-c $3.37(0.98) \mathrm{mmol} / \mathrm{L}$. The unadjusted proportion of patients achieving LDL-c target was $43 \%$ of intervention group, vs. $18 \%$ of controls $(x 2(1)=7.24, p<0.007)$. Intervention group subjects had a greater reduction in LDL-c $(1.59 \mathrm{mmol} / \mathrm{L}, \mathrm{SE} 0.15) \mathrm{vs}$. control (0.42 mmol/L, SE 0.10), $\mathrm{p}<0.0001$.

CONCLUSIONS: Pharmacist prescribing and follow-up in patients with dyslipidemia resulted in $>2$-fold more patients achieving recommended target LDL-c levels. This could have major implications for prevention of cardiovascular disease in Canada. 


\section{Pharmacists' perceptions of prescribing in two Canadian jurisdictions}

Lisa Guirguis, BScPharm, MSc, PhD; Lisa Dolovich, BScPhm, PharmD, MSc; Christine Hughes, BScPharm, PharmD, FCSHP; Mark Makowsky, BSP, PharmD; Cheryl Sadowski, BScPharm, PharmD; Nese Yuksel, BSCPharm, PharmD, FCSHP, NCMP

OBJECTIVES: In Canada, implementation and adoption of pharmacist prescribing varies between provinces. Our objective is to explore pharmacists' perceptions about prescribing between those who were currently prescribing (in Alberta) and those preparing to prescribe (in Ontario).

METHODS: $\quad$ Semi-structured, qualitative interviews (individual and group) explored views and experiences of pharmacists with or without prescribing authority. Pharmacists working in a variety of practice settings were selected using a mix of random and purposive sampling. Investigators analyzed each transcript using a qualitative approach to identify themes. Themes arising in the two jurisdictions were analyzed for similarity and differences.

RESULTS: In 2010, thirty-eight pharmacists in Alberta and 11 pharmacists in Ontario were interviewed and 37 pharmacists in Ontario participated in three focus groups. All pharmacists identified issues related to liability, impact on relationships with other health care professionals, practice environments and further training. Pharmacists preparing to prescribe were more likely to express concerns related to liability. Pharmacists with prescribing experiences were more likely to stress the importance of physician relationships, increased comfort with prescribing, report that prescribing legitimized prior practices and were less likely to prescribe if they believed patients should share responsibility for ensuring continuity of medication therapy.

CONCLUSIONS: By focusing on two jurisdictions at different stages of the adoption of prescribing, we found that pharmacists shared similar views on liability and the importance of physician relationships, continuing education and environmental support for prescribing. For pharmacists with prescribing experience, the importance of physician relationships intensified while concerns about liability were attenuated. 


\title{
Developing, implementing and evaluating deprescribing guidelines for the elderly as a mixed methods approach
}

\author{
Barbara Farrell, BScPhm, PharmD, FCSHP; James Conklin, PhD; Natalie Ward, PhD (ABD); Hannah \\ Irving, MA; Lalitha Raman-Wilms, BScPhm, PharmD, FCSHP; Lisa Mccarthy, BScPhm, PharmD, MSc; \\ Carlos Rojas-Fernandez, BScPharm, PharmD; Kevin Pottie, MD MCISc; Pamela Eisener-Parsche, MD; Lisa \\ Sunstrum, BSc, BScPhm (Candidate)
}

OBJECTIVES: This project aims to develop, implement and evaluate three deprescribing guidelines to assist clinicians in discontinuing medications that may no longer be indicated or may be causing side effects in elderly patients. The evaluation will provide an enhanced understanding of the way deprescribing guidelines, along with the processes of creating and implementing them, can influence behaviour change in clinical settings.

METHODS: $\quad$ Mixed methods including: modified Delphi consensus (expert meeting and surveys) to identify guideline priorities; developmental evaluation using ethnographic and case study analysis, observations and interviews with guideline development teams and implementation teams; descriptive analysis of guideline uptake and effect (chart audit); changes in clinician self-efficacy in deprescribing (survey); patient acceptance (interviews) and projected cost-savings from discontinued medications. Guidelines will be developed systematically by Guideline Development Teams and implemented into three Family Health Teams and three Long-Term Care sites.

RESULTS: $\quad$ Anticipated project outputs: development of feasible, effective deprescribing guidelines, an adaptable deprescribing implementation process for use with other drugs, better understanding of how deprescribing guidelines might bring about behaviour change and development of an outcomes evaluation approach to determine clinical and economic impacts.

CONCLUSIONS: By creating and optimizing a deprescribing guideline development and implementation process, this project aims to facilitate clinicians' ability to reduce inappropriate medication use. Input regarding effective deprescribing guideline implementation processes and relevant outcomes important to primary health care practitioners and researchers will be sought during the poster session. 


\section{Completeness of medication information in the outpatient referral process: a case for pharmacist involvement}

Barbara Farrell, BScPhm, PharmD, FCSHP; Barry Power, BScPhm, PharmD; Corey Tsang, BSc, BScPhm (Candidate); Clare Liddy, BSc, MD, MSc, CCFP, FCFP; Erin Keely, MD, FRCPC; Cynthia Way, BScPhm

OBJECTIVES: No studies have specifically addressed the completeness of medication information included in specialist referral or consultation documents. This study aims to determine completeness of medication information in

a) referral letters by primary care practitioners (PCP) provided to endocrinologists and

b) subsequent consultation notes.

METHODS: $\quad$ A retrospective chart audit was completed October-December 2013 in The Ottawa Hospitals endocrinology and metabolism clinic. Referral letters and consultation notes were accessed using the clinics electronic medical record with ethics approval. Medication lists were considered complete if they contained medication name, dose and frequency; completeness was also examined in consultation notes by assessing whether medication changes were communicated clearly.

RESULTS: $\quad 44 \%$ (31/70) of referral letters contained a medication list in the referral letter or supporting document. Of referrals containing a medication list, 71\% (22/31) were considered complete. $96 \%$ (67/70) of consultation notes contained a medication list of which $48 \%$ (32/67) were complete. 46\% (32/70) of consultation notes contained a medication change; in 38\% (12/32) it was unclear whether the change had been implemented or was a recommendation. Median time between referral and consultation was 105 days.

CONCLUSIONS: Medication information is present in referrals less than half of the time. When medication lists are present in referrals and consultation notes, information is frequently incomplete. Combined with the delay between referral and consultation, as well as lack of clarity regarding medication changes, these results build a case for investigating mechanisms to involve a pharmacist in the primary care/specialist referral process to facilitate medication reconciliation. 


\section{Impact of an online program to improve pharmacists' skills and confidence in providing patient care}

Barbara Farrell, BScPhm, PharmD, FCSHP; Douglas Archibald, MA ED, PhD; Corey Tsang, BSc; Lisa Pizzola, BASc, MSc

OBJECTIVES: ADAPT is an online continuing education program consisting of seven modules designed to enhance pharmacists' patient care and collaborative skills. Pilot results demonstrated learner satisfaction, increased confidence in performing skills and gains in knowledge along with intention to change practice. Program uptake has been significant across Canada. This study will determine if skills and confidence gains have been maintained and translated into adoption of expanded scope of practice activities and billable patient care services, improved quality of patient care and professional growth.

METHODS: $\quad$ The study uses a mixed methods approach, employing a survey, complemented with telephone interviews. Participants include Canadian and American pharmacists who have completed the ADAPT program since its inception in 2011 (approximately 240). Survey analyses use descriptive and comparative analyses with a content analysis of open-ended responses. Interview participants will be purposely selected from survey respondents. Interviews will be conducted using a semi-structured approach, audiotaped, transcribed and analyzed using a constant comparative approach.

RESULTS: $\quad$ Data collection and analysis will take place from January to March 2014. The experience of graduates attempting to employ new patient care skills in practice will be identified.

CONCLUSIONS: Findings will provide insight into pharmacists' ability to use knowledge and skills in adopting practice change. Barriers and other facilitators of interest to managers and policy makers will be identified. Researchers will benefit from an enhanced understanding of pharmacists' opinions about how their gains in skills and confidence have impacted on patient care. 


\section{6-2013 trends in adherence to} antidepressants, cardiovascular disease medications and diabetes medications in a community pharmacy

John Shaske, RPh; Mary De Vera, PhD; Carlo Marra, PhD; David Blackburn, PhD; Jim Danahy, BAA; George DeWolf, BSC

OBJECTIVES: To describe trends in adherence to antidepressants, cardiovascular disease medications and diabetes medications from 2006 to 2013 at the Howe Sound Pharmacy (HSP). HSP regularly performs medication management services and provides a natural setting for observational studies of medication adherence in real-world practice.

METHODS: We conducted a retrospective analysis of electronic pharmacy dispensation records from the HSP, using Adherence Track software. We used Anatomical Therapeutic Chemical (ATC) codes to identify patients with prescriptions for antidepressants, cardiovascular disease medications and diabetes medications between 2006 and 2013. First year adherence was calculated on newly initiated chronic medications defined with a one-year washout. Continual adherence was measured using all (including 1st time) dispensations for the target medication class in the year of assessment. The medication possession ratio (MPR) was calculated as the measure of adherence with a cut-off of 0.80 to categorize adherent and non-adherent patients.

RESULTS: $\quad$ Over the study period, we identified 14,034 patients and 1,155,846 dispensations. Overall, optimal adherence was observed in $43 \%$ and $64 \%$ of 1 st year and continual therapies, respectively. Adherence during 1st year therapies did not change over time. For continual therapies, adherence rates overall increased 31\% in 2006 to $79 \%$ in 2013. The same trends were observed with individual drug classes.

CONCLUSIONS: Adherence to continual therapy with antidepressants, cardiovascular disease medications and diabetes medications appears to have increased between 2006 and 2013 without changes in first year adherence. Further research is needed to identify factors associated with these findings. 


\section{A community pharmacy-based point of care lipid screening program to improve cardiovascular disease risk management}

John Papastergiou, BScPhm; Chris Folkins, PhD, BScPhm; Peter Tolios, BScPhm; Wilson Li, BScPhm, CDE; John Zervas, PharmD

OBJECTIVES: As Canada's most readily accessible health care providers, community pharmacists are uniquely positioned to assist patients in the assessment and management of cardiovascular disease (CVD) risk. Furthermore, recent changes to the scope of practice in Ontario permit pharmacists to sample patient's blood for the purpose of point of care (POC) screening. Here we describe a pilot initiative to provide POC lipid screening and Framingham Risk Score (FRS) assessment in a retail pharmacy setting, with the goal of helping patients to better understand and manage their CVD risk.

METHODS: LDL, HDL, total cholesterol, triglycerides, blood pressure and FRS were assessed at baseline in 56 patients. Interventions such as drug therapy changes and lifestyle modification were made as required. Follow-up screening was completed after 6 months in the 27 patients that were initially identified as moderate or high risk.

RESULTS: Of the 56 patients screened at baseline, 25 had clinically significant drug therapy problems which required a modification to their medication regimen. Recommended medication adjustments were communicated to the prescribing physicians for these 25 patients and in 9 cases responses were received indicating that the physician had implemented the pharmacist's recommendation. Patients in the follow-up group showed a trend towards increased mean HDL and decreased mean LDL, total cholesterol (TC), TC/HDL ratio, systolic blood pressure and 10-year CVD risk.

CONCLUSIONS: The apparent frequency of drug therapy problems in our patient population combined with our level of success in detecting them speak to the potential for simple, rapid community pharmacist-directed POC screening and consultation to assist patients in improving management of their CVD risk. 


\section{Chronic pain stakeholder engagement workshop}

Feng Cheng, BScPhm, PharmD; Ayesha Khan, BSc, MPH; Tejal Patel, BScPhm, PharmD; Kelly Grindrod, BScPharm, PharmD, MSc; Kay Aoki, BScPharm, PharmD (Candidate); King Chen, BSc, PharmD (Candidate); Ana Milic, PharmD (Candidate); Kathryn Mercer, BA, MI; Beth Sproule, BScPhm, PharmD

OBJECTIVES: To identify and better understand the challenges and opportunities for community pharmacists in optimally managing chronic pain as perceived by patients, caregivers, advocacy groups and health care providers, in order to plan for patient-centered pharmacist interventions.

METHODS: Health care professionals and advocacy groups working with chronic pain patients in the Southwest Ontario area were invited to participate via email or telephone. Patients and family members or caregivers were invited to participate through referral by health care professionals or the advocacy groups. Participants were assigned to one of three groups: professionals, advocates and patients/caregivers. Nominal Group Technique (NGT) was used to gather and rank data collected.

RESULTS: $\quad$ Seventeen (17) participants generated 69 items in total. Items were classified into 10 priority areas: education, trust, finance, scope of practice, health promotion, communication, access, feedback, team and technology. Education was identified as one of the top three challenges by all groups. Recognition of pharmacists as a member of the interdisciplinary team and improved communications among providers were also ranked as essential.

CONCLUSIONS: Participants identified numerous opportunities for the pharmacist to meet needs of patients with chronic pain. Results will be used to inform intervention design in the next phase. Part of Ontario Pharmacy Research Collaboration (OPEN). 


\title{
ClereMed: A rapid prototyping project to build a mobile screening tool to identify adults 55 and over who have difficulty reading medication labels
}

\begin{abstract}
Kelly Grindrod, BScPharm, PharmD, MSc; Allison Gates, MSc, PhD Student; Susan Leat, PhD; Lisa Dolovich, PharmD; Roderick Slavcev, PhD, MBA; Rob Drimmie; Behzad Aghaei, MASc; Calvin Poon, BScPharm; Shamrozé Khan, OD
\end{abstract}

OBJECTIVES: $\quad$ To determine the usability and reliability of a mobile application designed to identify adults 55 and over who are having difficulty reading and/or understanding prescription labels.

METHODS: ClereMed is an HTML-5 prototype application that simulates medication taking. Adults (55 years) were recruited through pharmacies, retirement residences and a low vision optometry clinic. Participants tested ClereMed using an iPad and also provided information on demographics, computer literacy and usability. Cognition was assessed using the Montreal Cognitive Assessment tool and functional reading ability was measured using the MNRead Acuity Chart. Participants also completed a real-life medication taking simulation using prescription vials, tablets and pillboxes.

RESULTS: $\quad$ For the 47 participants, $60 \%$ were female and the mean age was $76 \pm 11$ years. Only four participants were recruited and screened by pharmacists. A third did not own a computer or touchscreen device. The mean usability score was 76/100. ClereMed correctly identified $71 \%$ of participants with functional reading difficulty but only $21 \%$ participants with cognitive impairment. Participants who owned a computer/ touchscreen completed ClereMed in a mean time of 26( \pm 16$)$ seconds, compared to $52( \pm 34)$ seconds for those who do not own a device $(p<0.001)$. Those who had difficulty struggled with screen glare, button activation and the "drag and drop" function.

CONCLUSIONS: ClereMed was well accepted by older participants. It may be most useful as a tool to help family members and caregivers identify adults 55 and over with impaired functional reading ability. Future research will focus on improved sensitivity for measuring cognitive impairment and on the feasibility of implementing a mobile application into pharmacy workflow. 


\section{Community pharmacists' attitudes toward dispensing errors at community pharmacy settings in Central Saudi Arabia}

Mohamed Al-Arifi, PhD

OBJECTIVES:

The main objective of this study was to survey pharmacists' attitudes toward dispensing errors in community pharmacy settings in Saudi Arabia.

METHODS: $\quad$ A cross-sectional survey of community pharmacists in Riyadh region, Saudi Arabia was conducted over a period of 6 months from March through September 2012. A stratified random sample of 800 registered pharmacy practitioners was collected all over Riyadh region. Statistical analysis was done using SPSS version19.0 for windows (SPSS Inc., Chicago, Illinois).

RESULTS: $\quad$ The response rate was almost $82 \%$. The majority of the respondents are young adults (90.2\%). The median for years of registration of respondent pharmacists was 9 years (range 1-37 years). About 62\% (407) of the respondents have a positive response while only $37.8 \%(n=248)$ have a negative response in this respect. The major factors identified were pharmacist assistant (82.2\%) and high workload (72.5\%). The most appreciated factors that help reducing dispensing errors are improving doctor's hand writing and reducing work load of the pharmacist ( $82.9 \%$ and $82.8 \%$ respectively), having drug names that are distinctive (76.1\%) and having more than one pharmacist in duty (75.5\%).

CONCLUSIONS: In conclusion, majority of community pharmacists indicated that the risk of dispensing errors was increasing and most of them were aware of dispensing errors. It is obvious from the study results that dispensing errors are a big concern for community pharmacy practice in Saudi Arabia. Therefore, there is an urgent need for the professional organizations and Pharmacy Boards in Saudi Arabia to determine standards for the profession. 


\title{
Consumers' evaluation of community pharmacy services in Al-Ahsaa province, eastern region of Saudi Arabia
}

\author{
Mohammad Zaitoun, BPharm, PharmD; Ali Alyounis, PharmD; Ahmed Al-Naim, PharmD
}

OBJECTIVES:

Community pharmacies play a major role in health systems. We wanted to investigate consumers' evaluation of community pharmacy services in Al-Ahsaa province, in the eastern region of Saudi Arabia.

METHODS: $\quad$ Cross-sectional study was conducted from April 22 to May 25, 2013, among a sample of consumers. A self-administered questionnaire was designed (Cronbach's Alpha $=0.76$ ). Three items were about demographics. Thirteen items focused on patient education. For this part, Likert score was calculated. Six items assessed pharmacists' behavior, pharmacy appearance, drug availability, views towards pharmacists as information providers and general evaluation of services. Rating for this part was presented as very good, good, weak, very weak and not sure.

RESULTS: $\quad$ From the participants ( $n=387$ ), 46\% rated services in general as good, 26\%, 26\% and $2 \%$ rated it as Very Good, Weak and Very Weak. Respectively, 59\% of participants considered physicians as the best drug information providers. For the patient education sessions, 28\% stated that pharmacists always dispense their medications without asking any questions and $27 \%$ reported this as frequent, the average score was 35.2 out of 65, with main defects in the following components: Action after missed dose, medication history, storage conditions, drug interactions and onset of action. (Never explained $=53,46,44,4$ and $37 \%$ respectively) Forty-five percent of the participants rated pharmacists' responses to their inquiries as Very Good and 34\% as Good. Sixty Five percent evaluated pharmacists' behavior as very good and 33\% as Good. For the general appearance of pharmacy, 68\% rated it as Very Good and $25 \%$ as Good. Forty percent evaluated the drugs availability as very good while $36 \%$ rated it as good.

CONCLUSIONS: Efforts are needed to increase consumers' awareness about community pharmacists' role as information providers and eliminate any possible causes that hinder effective patient counseling. 


\section{Continuous quality assurance pilot project in Saskatchewan community pharmacies}

Certina Ho, RPh, BScPhm, MISt, Med; Jim Kong, BSc, PharmD (Candidate); Carol Lee, CHIM

OBJECTIVES: Vulnerabilities in health care system processes can lead to medication errors that can result in patient harm. A Continuous Quality Assurance (CQA) pilot project was implemented in Saskatchewan to determine underlying system-based contributing factors to near misses or medication incidents in community pharmacies.

METHODS: $\quad$ Since its implementation in October 2013, 3.4\% (11) of 328 community pharmacies in Saskatchewan have participated in this pilot project. They include chain, retail and independent pharmacies. From October 2013 to January 2014, 123 medication incidents have been voluntarily reported to ISMP Canada. The incidents were analyzed, with a focus on the severity of outcome of the incidents and medication-use areas associated with these incidents.

RESULTS: Of the 123 incidents reported, 82\% (101 of 123) were near misses and 18\% (22 of 123) resulted in no harm to the patient. The majority of incidents occurred during the prescription order entry, with the remainder occurring during prescription preparation or dispensing. The most common types of incidents reported were incorrect dose/ frequency (24\%), incorrect quantity (24\%) and incorrect prescriber (13\%). Cardiovascular medications such as anti-hypertensives were the most commonly reported, with various diabetic, anti-psychotic and asthma medications being frequently reported as well. Possible contributing factors of these incidents include interruptions in workflow, illegible prescription orders, dangerous abbreviations and look-alike/sound-alike drug names.

CONCLUSIONS: This CQA pilot project represents a novel method of addressing medication incidents and improving patient safety in the community pharmacy setting. Through the analysis of incidents and sharing of findings, pharmacy practitioners can learn from reported incidents and implement safeguards. 


\section{Drug shortages and patient safety}

Certina Ho, RPh, BScPhm, MISt, Med; Lindsay Yoo, RPh, BSc, BScPhm; Calvin Poon, RPh, BSc, BScPhm, ACPR; Marvin Ng, BSc, PharmD (Candidate); Kathy Li, BSc, PharmD (Candidate); Roger Cheng, RPh, BScPhm, PharmD

OBJECTIVES: $\quad$ The issue of ongoing drug shortages has become a significant challenge to pharmacists, patients and prescribers. Drug shortages have been shown to delay important drug therapy and result in medication errors. This project aims to identify themes associated with drug shortage related incidents and illustrate important considerations in the management of drug shortages in community pharmacy practice.

METHODS: $\quad$ An analysis of medication incidents related to drug shortage was performed using reports anonymously submitted to the Institute for Safe Medication Practices Canada (ISMP Canada) Community Pharmacy Incident Reporting (CPhIR) Program from November 2010 to June 2012.

RESULTS: $\quad$ A total of 62 reports were included in the analysis. Several themes of drug shortage related incidents were identified including risk of overdose/underdose, wrong drug, incorrect brand or strength selected, as well as patient confusion and misunderstanding of the altered medication. Two major points in the management of drug shortages have been illustrated:

1) It is important to perform independent double checks during the order entry and dispensing process when making alternative arrangements for a prescription;

2) There is the need for patients to understand the identity and use of the altered medication through prescription counseling, monitoring and follow-up.

CONCLUSIONS: Drug shortages continue to be an inevitable issue that often led to negative impacts on patient safety. Community pharmacists can mitigate and prevent the likelihood of negative outcomes from occurring through understanding the common themes and important management concepts associated with drug shortages. 


\section{Effectiveness of extracurricular journal clubs on pharmacy students' learning of evidence-based medicine and critical appraisal}

Certina Ho, RPh, BScPhm, MISt, Med; Joyce Tsang, BScPhm (Candidate); Olla Wasfi, MSc, PhD; Mary Power, PhD; Brett Morphy, RPh, BSc, BScPhm; Saurabh Patel, RPh, BScPhm; Calvin Poon, RPh, BSc, BScPhm, ACPR; Boris Tong, RPh, BSc, BScPhm

OBJECTIVES:

Pharmacists are medication therapy experts. It is important to fully equip prospective pharmacists early in their undergraduate training on how to practice evidence-based medicine (EBM) and critical appraisal (CA) skills. This study intends to investigate the effectiveness of extracurricular student-driven journal clubs in enhancing pharmacy students EBM knowledge and CA of primary literature.

METHODS: $\quad$ Eight one-hour extracurricular journal club sessions were organized by students. Students who voluntarily attended journal clubs were asked to complete an online questionnaire to self-report their learning and understanding of EBM and CA concepts. Journal club presenters were invited to focus groups to share their experience.

RESULTS: $\quad$ Attendance of each journal club ranged from 25-50 students. Out of the 28 students who completed the questionnaire, $57 \%$ agreed that they were able to critically appraise primary literature in a timely fashion; $68 \%$ believed that they were able to formulate clinically-relevant conclusions from research studies; and 57\% were confident in presenting clinical decisions based on assessment of a research study. We conducted 3 focus groups with 22 student presenters who found themselves more proactive in seeking evidence-based clinical decisions and strived for continuous development of their CA skills.

CONCLUSIONS: Pharmacy students self-reported that journal clubs offered them a platform to further practice and apply their knowledge on EBM and CA skills. Traditional didactic teaching/learning in classroom serves as an introduction to EBM and CA. Students need to continuously practice these skills in order to fulfill the competencies of being a medication therapy expert capable of evaluating and applying EBM in practice. 


\section{Handbook for a pilot study to reduce potential hospitalizations due to preventable drug-drug interactions}

Certina Ho, RPh, BScPhm, MISt, Med; Atsushi Kawano, RPh, BSc, MSc, BScPhm

OBJECTIVES: Hospital reports on medication incidents suggest 37\%-51\% of reported adverse drug events, including drug-drug interactions (DDIs), may have been prevented with appropriate interventions. This study intends to compile a list of evidence-based DDIs with association to an increased risk of hospitalizations and develop a treatment algorithm handbook to facilitate pharmacists or clinicians in ambulatory care in identifying and offering recommendations to prescribers to prevent these DDIs.

METHODS: $\quad$ A comprehensive literature search was conducted and articles were selected based on relevant DDIs that were associated with an increased risk of hospitalization. Evidencebased treatment algorithms were created to suggest alternative therapeutic options for three common community infections-Group A B-hemolytic Streptococcus pharyngitis, outpatient community-acquired pneumonia and uncomplicated lower urinary tract infections.

RESULTS: $\quad$ Evidence-based DDIs identified in this study involved either a macrolide or trimethoprimsulfamethoxazole. In all cases, the evidence supported an alternative to either antibiotic for selected community infections. Older persons were underrepresented in trials evaluating antibiotic therapy for community infections. Selecting an appropriate antibiotic required using data derived primarily from children and adults. A treatment algorithm handbook was created for clinicians in ambulatory care.

CONCLUSIONS: The list of evidence-based DDIs with association to an increased risk of hospitalizations identified in this study was made available to all pharmacists via the Ontario College of Pharmacists quarterly publication, Pharmacy Connection, in Spring 2013. Pharmacists/ clinicians have the option of using the treatment algorithm handbook developed in this project to help resolve and prevent these DDIs. 


\section{Methadone medication incidents in community pharmacy}

Certina Ho, RPh, BScPhm, MISt, Med; Atsushi Kawano, RPh, BSc, MSc, BScPhm; Dilpreet Bhathal, RPh, BScPhm; Jim Kong, BSc, PharmD (Candidate); Roger Cheng, RPh, BScPhm, PharmD

OBJECTIVES: In community pharmacy practice, methadone medication incidents can occur at any stage of the medication use process and carry serious harmful implications for the patients involved. This study aims to identify system-based contributing factors that facilitate these medication incidents.

METHODS: $\quad$ A qualitative, multi-incident analysis was conducted on 72 incidents, anonymously reported to ISMP Canada between April 2010 to August 2012, which involve Methadone and/or Metadol. All incidents were reviewed independently by two ISMP Canada analysts and categorized into themes and subthemes of contributing factors.

RESULTS: $\quad$ Most of the incidents reported involved the Methadone Maintenance Treatment (MMT) program, which utilizes oral methadone for opioid addiction. The two main themes realized from this study were the medication-use process of methadone, as well as characteristics that were unique to methadone. The subthemes included the various stages of the medication use process such as prescribing, order entry, dispensing and administration. Two of the most significant contributing factors that had a positive correlation with methadone incidents were the highly individualized dosing associated with MMT and the lack of independent double checks.

CONCLUSIONS: The use of methadone as a potent anti-addictive and analgesic opioid continues to present challenges when adopted by community pharmacies. Its highly individualized dosing regimen, along with its complex dispensing process, can undermine its value as a therapeutic option if medication incidents continue to occur. This study provides applicable recommendations for pharmacy practitioners to improve the medication use process of methadone and ultimately enhance medication safety. 


\section{Multi-incident analysis of medication incidents involving contraceptives}

Certina Ho, RPh, BScPhm, MISt, Med; Tomasz Lechowicz, BSc, BScPhm (Candidate); Lindsay Yoo, RPh, BSc, BScPhm; Kathy Li, BSc, PharmD (Candidate); Roger Cheng, RPh, BScPhm, PharmD

OBJECTIVES: Contraceptives are unique from other medications, as they are available in a wide array of packages to accommodate for different dosing regimens. Medication incidents involving contraceptives can occur at various medication use stages. This research project aims to identify themes and corresponding contributing factors of medication incidents associated with contraceptives in community pharmacy practice.

METHODS: $\quad$ An analysis of medication incidents related to contraceptives was performed using reports anonymously submitted to the Institute for Safe Medication Practices Canada (ISMP Canada) Community Pharmacy Incident Reporting (CPhIR) Program from November 2009 to March 2013. This analysis was conducted using a search criterion of the brand names of approved contraceptives in Canada.

RESULTS: $\quad$ A total of 256 reports were included in the analysis. Major themes of medication incidents involving contraceptives were identified, including look-alike/sound-alike drug names, incorrect quantity, incorrect directions of medication use, insufficient documentation of patient medication history, lack of patient education and brand/generic names confusion. These errors can occur at various points along the stages of medication use, such as prescribing, order entry, preparation/dispensing and administration.

CONCLUSIONS: The common themes contributed to medication incidents involving contraceptives identified in this study may be extrapolated to other therapeutic areas in pharmacy practice. Pharmacists can apply incident analysis findings to pinpoint specific areas of weakness in their medication delivery systems and to prevent risk in daily practice. The ultimate goal is to continuously improve patient safety and thus the quality of care through the analysis of incidents and sharing of lessons learned from these reports. 


\section{The role of online/smartphone applications in Type II Diabetes Management: A qualitative study}

Certina Ho, RPh, BScPhm, MISt, Med; Tomasz Lechowicz, BSc, BScPhm (Candidate); Lindsay Yoo, RPh, BSc, BScPhm; Kathy Li, BSc, PharmD (Candidate); Roger Cheng, RPh, BScPhm, PharmD

OBJECTIVES: Multiple interventions, including smartphone applications (or apps), have been developed for patients to self-manage diabetes. This study intends to identify a list of smartphone applications that can be recommended to patients for self-management of diabetes with respect to medication adherence, physical activity, diet and weight management.

METHODS: $\quad$ An environmental scan was performed to identify and evaluate the top 7 diabetes management apps for iPhone, iPad, iPod Touch android and Windows Phones. These apps were assessed based on features, usability and their authority, accuracy, currency, objectivity and quality. We interviewed 4 Certified Diabetes Educators (CDEs) and obtained their feedback and experience on the use of these apps in diabetes patient education.

RESULTS: $\quad$ The assessment of the 7 apps revealed some key features that were available in some but not others, medication reminders; tracking of blood glucose readings, insulin dosing, physical activity, weight, blood pressure readings and carbohydrate intake; electronic synchronization with health care providers and glucometer compatibility. We identified that simplicity of the app, ease of use and cost were the key factors in determining the best app for self-management of diabetes. iBG Star and Glucose Buddy both fulfilled these criteria and Tactio Health was a close second.

CONCLUSIONS: Smartphones are now an integral part of everyday life. Patients play a critical role in chronic disease management. Pharmacists can expect to receive questions about the role of smartphone apps in the management of diabetes. When recommending phone apps to diabetic patients, it is important to individualize app selection to ensure optimal benefits to patient care. 


\section{Controversy vaccination}

Shawn Bugden, BScPharm, MSc, PharmD; Kevin Friesen, BScPharm

OBJECTIVES: As the scope of practice across the country expands to include the administration of vaccines, pharmacists move even closer to the front line of the vaccine controversy. Andrew Wakefield's discredited research linking autism and vaccination casts a long shadow. Vaccination remains an area of controversy and pharmacists have a role in dispelling myths and providing accurate information to their patients. In order to fulfill this role, pharmacists must understand the controversy and the facts. An educational session was provided to Manitoba pharmacists as a knowledge booster to enhance their protection against the controversy. Our objective was to determine the impact of this session on pharmacists' knowledge of the vaccination controversy.

METHODS: $\quad$ Pharmacists filled out a survey examining their knowledge of autism/vaccine controversy pre and post a professional development session. Baseline knowledge was compared with the survey results after the educational session. Results were reviewed in the aggregate.

RESULTS: $\quad$ On average, correct responses increased significantly by $23 \%$ overall, from $68 \%$ to $91 \%$ $(P<0.01)$. The questions with the lowest initial correct answer rate showed the greatest improvement. Questions related to thiomersal, vaccination rates and the biology of autism showed the greatest degree of improvement.

CONCLUSIONS: While baseline knowledge of pharmacists was high in some areas, some of the more subtle concepts were not well understood. This evaluation suggested that the knowledge of the controversy was enhanced by the professional development session. It is hoped that pharmacists with this knowledge will have some protection when the controversy presents at their counter. 


\section{To crush or not to crush}

Shawn Bugden, BScPharm, MSc PharmD; Peter Stockmann, BScPharm (Candidate);

Kevin Friesen, BScPharm

OBJECTIVES: The United States, United Kingdom and Australia have developed resources to guide health care professionals when considering altering solid dosage forms. Since medications and their trade names are country specific, Canadian reliance on outside resources to guide crushing decisions is problematic. Our objective was to create a comprehensive Canadian resource to guide these decisions.

METHODS: $\quad$ A literature search was conducted to discover determine the major resources used to guide crushing. We cross referenced these resources to create a comprehensive list of target products. This list was supplemented with drugs identified as hazardous by $\mathrm{NIOSH}$. All identified products were then screened through Canadian documents (CPS, Minor Ailments). Supplementary information was obtained from published literature and drug manufacturers as required.

RESULTS: $\quad$ The resulting Canadian Do Not Crush List included 211 active ingredients identified as having formulations that should not be crushed, or that require special instructions in order to do so. A rationale was given for the inclusion of each medication on the list. Practical solutions for patients with swallowing difficulties were provided where possible. A condensed list was also created to support nursing and other allied health professionals.

CONCLUSIONS: This project has created a Canadian Do Not Crush List. It is expected that maintenance and continuous quality improvement will be an ongoing and iterative endeavour. We introduce this list to the Canadian pharmacist community and hope the document provides a system to facilitate interprofessional collaboration between pharmacists and those who administer medications and that this may address the risks associated with altering solid-dose oral formulations. 


\section{Developing a Recognition of Prior Learning (RPL) process for implementation in a PharmD bridging program}

Rosemary Killeen, RPh, BScPhm; Nancy Waite, PharmD, FCCP; Eric Schneider, PharmD

OBJECTIVES: To give academic credit for clinical competencies acquired through work experience and formal training, the PharmD bridging program for UWaterloo BScPhm graduates includes a Recognition of Prior Learning (RPL) process. RPL is used to systematically validate skills, knowledge and abilities and give credit for prior learning. No existing RPL model was found in Canadian pharmacy programs, so an investigative process was used to gather evidence for the RPL design. Key factors included best practices, academic rigour, consistency with in-house PharmD program assessments, accreditation standards, feasibility and resources.

METHODS: $\quad$ A review of existing Canadian and international resources was conducted. The Canadian Association of Prior Learning Assessment conference provided background to the process. Personnel at institutions utilizing RPL in health care training shared expertise via phone interviews. A summary was prepared and used to develop the RPL template.

RESULTS: $\quad$ A two-part process for the bridging program was developed to capture students' level of clinical competence and eligibility to receive credit for prior learning. In part one, students work up patient cases, which are assessed for problem solving abilities orally and in writing. This allows for measurement of complex, cognitive learning and facilitates concurrent evaluation of competencies. In part two, students complete a clinical portfolio, documenting competence in various learning outcomes. These are reviewed and graded by trained assessors. This process was approved for implementation in the 2015 launch of the bridging program.

CONCLUSIONS: A valid, rigorous and reliable RPL process can be developed to recognize pharmacists' prior learning for academic credit at the PharmD level. 


\section{Development of an interdisciplinary student managed, primary health care clinic in Scotland: A Canadian collaboration}

Anita Weidmann, MPharm, MRPharmS, FHEA, PhD; Vibhu Paudyal, BPharm, SRPharmS, PhD; Alison Strath, BSc(Pharm), FRPharmS; Derek Jorgenson, BSP, PharmD

OBJECTIVES: To identify the local need in Aberdeen, Scotland, for a health care student led, outof-hours walk in service and to explore the general public's expectations from such a health initiative?

METHODS: $\quad$ A cross-sectional postal questionnaire survey was used. 3000 randomly selected members of the general public in city and shire aged 18 years and over were sent a postal questionnaire. Two reminders were sent at two-weekly intervals. The questionnaire explored current use of the health care system; participant views on a health care student led, walk-in service and their personal opinion on the availability of such a service.

RESULTS: Questionnaires were returned by $27.5 \%(n=824)$. Over half of respondents (62.4\%; $\mathrm{n}=514$ ) would consider accessing health care from a student led, walk in service. Younger respondents (aged 18-29) were more likely to consider accessing the student led walk in service (70.6\%; $\mathrm{n}=24$ ) compared with older (aged 60 or over) respondents (49.5\%; $n=253)(p=0.002)$. The range of services they expect to see include general health checks (60\%; $n=494$ ); smoking cessation (54.4\%; $n=448$ ); weight management (56.8\%; $n=468$ ); drug misuse services (47.2\%; $n=387$ ) and help for sexually transmitted diseases ( $57.5 \%$; $n=474$ ). The majority of respondents had a positive attitude toward such a service (62.4\%; $n=514)$.

CONCLUSIONS: The establishment of inter-professional student led clinic across Canada has become a flagship model of teaching an integrative approach to pharmacy students. The need for the first UK-based clinic in Aberdeen has been established with this project. 


\section{Pharmacist prescribing education: An international knowledge exchange study}

Brian Addison, MPharm; MRPharmS; Anita Weidmann, MPharm, MRPharmS, FHEA, PhD; Diane Harpell, BScPharm, MBA; Harriet Davies, BScPharm, CDE, MEd

OBJECTIVES: Pharmacists in Nova Scotia have recently expanded their scope of practice to include prescribing for patients with a variety of minor conditions, such as dyspepsia, smoking cessation and cough (2010). In Scotland, pharmacists have had prescribing rights since 2003. Mandatory training of 26 days in higher education and 12 days of learning in practice under the mentorship of a physician is an essential requirement for all pharmacist prescribers in Scotland. To carry out a pilot study allowing a group of Canadian pharmacists to partake in the higher education training provided for independent pharmacist prescribers in Scotland.

METHODS: A complex collaborative project between the Robert Gordon University, Aberdeen, and Dalhousie University, College of Pharmacy, Halifax, NS.

RESULTS: $\quad$ Five pharmacists from Nova Scotia attended a one week residential period at Robert Gordon University, School of Pharmacy, Aberdeen in June 2013. In addition pharmacists had access to all online materials and spent one day in practice with an already established independent pharmacist prescriber. Any area of practice overlap between the two countries will be used to inform pharmacist prescribing training in Canada.

CONCLUSIONS: This is a prime example of how innovative pharmacy practices can be lead to knowledge transfer and improved pharmacy education across international borders. 


\section{Pay-for-performance remuneration for pharmacist prescribers' management of hypertension: A pre-specified sub-study of the Alberta Clinical Trial in Optimizing Hypertension (RXACTION)}

Sherilyn Houle, BSP, PhD; Ross Tsuyuki, BScPharm, PharmD, MSc, FCSHP, FACC; Theresa Charrois, BScPharm, ACPR, MSc; Michael Kolber, BSC, MD, CCFP, MSc; Finlay McAlister, MD, MSc; Meagen Rosenthal, BA, MA, PhD (Candidate); Richard Lewanczuk, MD, PhD; Dale Cooney, BScPharm

OBJECTIVES: $\quad$ Pharmacy practice is shifting from a focus on distribution to direct patient care. To be sustainable, pharmacists must receive appropriate payment for these services. This pre-specified sub-study of the RXACTION study aimed to determine if blood pressure (BP) lowering differed between intervention patients whose pharmacist was paid by fee-for-service (FFS) or pay-for-performance (P4P).

METHODS: $\quad$ Patients with elevated BP assigned to enhanced care were further randomized to P4P or FFS payment for the pharmacist. In FFS, pharmacists received $\$ 150$ for the initial visit and $\$ 75$ for follow-up visits. P4P included FFS payments plus incentives of $\$ 125$ and $\$ 250$ for each patient who reached $50 \%$ and $100 \%$ of the BP target, respectively. The primary outcome was difference in change in systolic BP between P4P and FFS groups.

RESULTS: $\quad$ A total of 181 patients were randomized to enhanced care, with 92 in the FFS and 89 in the P4P group. Patients average (SD) age was 63.5 (12.7) and 48.8\% were male. Three-quarters (77.8\%) were on antihypertensive drug therapy at baseline, taking 1.7 medications on average. Mean systolic BP reduction in the P4P and FFS groups were 19.0 (SD 17.0) and 16.4 (SD 17.1) $\mathrm{mm} \mathrm{Hg}$, respectively. The difference in change in SBP was $2.6 \mathrm{~mm} \mathrm{Hg}(\mathrm{p}=0.32)$. Due to early study termination, the pre-determined sample size (125 per group) was not achieved.

CONCLUSIONS: This trial of pharmacist prescribing found no appreciable difference in the magnitude of $\mathrm{BP}$ reduction achieved when pharmacists were paid by $\mathrm{P} 4 \mathrm{P}$ versus FFS, although both groups did exhibit substantial reductions in SBP. 


\section{Paying pharmacists for patient care: A systematic review of remunerated pharmacy clinical care services}

Sherilyn Houle, BSP, PhD; Kelly Grindrod, BScPharm, ACPR, PharmD, MSc; Trish Chatterley, MLIS; Ross Tsuyuki, BScPharm, PharmD

OBJECTIVES: $\quad$ Expansion of scope of practice and diminishing revenues from dispensing are requiring pharmacists to increasingly adopt clinical care services into their practices. To provide clinical care, pharmacists must be able to receive payment in order to be sustainable. This study updates our previous review by reporting on remunerated pharmacist clinical care programs worldwide and their uptake and patient care outcomes.

METHODS: Literature searches were performed in several databases including MEDLINE, Embase and International Pharmaceutical Abstracts for papers referencing remuneration, pharmacy and cognitive services. Programs were included if they were implemented prior to December 2012 and were not reported our previous review. One author performed data abstraction, which was independently reviewed by a second author. All results are presented descriptively.

RESULTS: $\quad$ Sixty new programs were identified across Canada, the United States, Europe, Australia and New Zealand, ranging in complexity from emergency contraception counseling, to minor ailments schemes and comprehensive medication management. In North America, the average medication review fee is $\$ 68.86$, with $\$ 23.37$ offered for a followup visit and $\$ 15.16$ for prescription adaptations. Time-dependent fees were reimbursed at $\$ 93.60$ per hour on average (all figures in $\$ C D N$ ). Few programs evaluated uptake and outcomes of these services but, when available, indicated slow uptake but improved chronic disease markers and cost-savings.

CONCLUSIONS: Remuneration for pharmacists' clinical care services is highly variable, with few programs reporting program outcomes. Programs and pharmacists are encouraged to examine the time required to perform these activities and outcomes achieved to ensure fees are adequate to sustain these patient care activities. 


\section{The validity of home blood pressure monitor validation studies}

Yazid Al Hamarneh, BScPharm, PhD; Sherilyn Houle, BScPharm, PhD; Trish Chatterley, MLIS; Ross Tsuyuki, BSCPharm, PharmD, MSc, FCSHP, FACC

OBJECTIVES: Recent guidelines highlight the importance of home blood pressure monitoring. Although validation studies of home monitors have been published, the quality of their methods has not been evaluated. To perform a systematic review of the published peer-reviewed literature on the validity of home blood pressure monitors and to assess their adherence to validation standards.

METHODS: $\quad$ We searched MEDLINE, Embase, Cochrane Library and CINAHL to April 2013. Studies were included if they claimed to evaluate the accuracy/validity of upper arm home blood pressure monitors and were excluded if conducted only in special populations (pregnancy, kidney disease, etc.) or using wrist or finger devices. Study methods were compared to the validation protocol(s) referred to in the article (e.g., British Hypertension Society) or, if not specified, to protocols existing at the time of publication.

RESULTS: $\quad 4473$ studies were identified; 97 met our inclusion criteria. Across all included studies, $93 \%$ reported comparing the test device with manual readings, $80 \%$ had a sufficient sample size, $79 \%$ utilized two trained observers, 68\% took an adequate number of measurements per subject, $67 \%$ took measurements following the proper sequence and only $15 \%$ described enrolling subjects of sufficient variability (age, systolic and diastolic BP and arm circumference).

CONCLUSIONS: None of the reviewed studies claiming to validate home blood pressure monitors fully adhered to the validation standards. This may lead to both false-positive (claims that the device is accurate) and false-negative claims. When interpreting the findings of validation studies, pharmacists should critically evaluate their adherence to accepted validation standards. 


\section{The design of the Alberta Vascular Risk Reduction Community Pharmacy Project: $\mathrm{R} \times \mathrm{EACH}$}

Yazid Al Hamarneh, BScPharm, PhD; Ross Tsuyuki, BScPharm, PharmD, MSc, FCSHP, FACC; Brenda Hemmelgarn, MD, PhD, FRCP(c); Charlotte Jones, MD, PhD, FRCP(c); Oladele Dunsi, PhD

OBJECTIVES: Risk factors for vascular disease remain poorly identified and treated. To evaluate the effect of a community pharmacy-based case finding and intervention program on reduction in cardiovascular risk.

METHODS: $\quad$ Design: Randomized controlled trial Setting: 70 community pharmacies in Alberta. Patients: 1180 adults at high risk for cardiovascular events (including high risk primary prevention, established vascular disease, chronic kidney disease and/or diabetes) identified by the pharmacist. Intervention: The pharmacist will conduct a structured medication review, including patient assessment, laboratory tests (HbAlc, lipid panel, creatinine, urine albumin), individual cardiovascular risk assessment and education regarding this risk. Pharmacists will prescribe, adapt, or recommend medications as necessary to achieve targets for lipids, smoking cessation, glycemic control and hypertension. Follow-up is every 4 weeks for 3 months. Usual care by the pharmacist and physician with no specific intervention for 3 months. After that, patients are crossed-over to receive the intervention described above and followed for another 3 months.

RESULTS: $\quad$ Primary outcome: Difference in change in cardiovascular risk between intervention and control groups. Cardiovascular risk is defined as the risk for future cardiovascular events (myocardial infarction, revascularization, cardiovascular death) as estimated by validated risk engines (Framingham, International and UKPDS).

CONCLUSIONS: This is the first large scale randomized trial on global cardiovascular risk reduction in a community pharmacy setting. It utilizes the community pharmacists expanded scope of practice and the existing remuneration system in Alberta. RxEACH is a unique collaboration between Alberta Health, Alberta Health Services, University of Alberta, University of Calgary and industry. 


\section{Economic analysis of the RxING study}

Yazid Al Hamarneh, BScPharm, PhD; Luc Sauriol, MSc Economics; Ross Tsuyuki, BScPharm, PharmD, MSc, FCSHP, FACC

OBJECTIVES: $\quad$ Many patients with type 2 diabetes (T2DM) require insulin treatment. Pharmacists are frontline health care professionals who see patients with T2DM frequently and as such, could help them achieve their targets by intervening in a timely manner. RxING showed that community pharmacist prescribing of insulin glargine in uncontrolled patients with T2DM resulted in an average A1c reduction of $1.8 \%(95 \% \mathrm{Cl} 1.4$ to 2$)$. To assess the costeffectiveness of pharmacists' early intervention in prescribing insulin in people with T2DM.

METHODS: $\quad$ We used the IMS CORE Diabetes Model, a Markov structure and Monte Carlo simulation model, to assess complications and disutilities and health utility and cost data were obtained from recent literature. The efficacy of insulin in terms of HbA1c reduction and rates of hypoglycemia were obtained from RxING. Base case analyses examined the effects of having pharmacist prescribe insulin 1, 2 and 3 years earlier than a physician.

RESULTS:

Having pharmacists prescribe insulin 1 year earlier than a physician resulted in an incremental cost savings of $\$ 790$ (CDN\$) and a gain of 0.048 QALYs per patient. Early pharmacist prescription of insulin by 2 years resulted in an increment cost savings of $\$ 687$ (CDN\$) and a gain of 0.075 QALYs per year. At 3 years earlier, the results were a cost savings of $\$ 105$ and a gain of 0.086 QALYS.

CONCLUSIONS: Having pharmacists initiate insulin sooner in uncontrolled T2DM results in cost-savings and would delay the development of diabetes-related complications resulting in improved quality of life and increased survival rates. 


\section{Pharmacist comfort with reporting quality-related events (QREs) of varying severity}

Todd Boyle, PhD; Thomas Mahaffey, PhD; Andrea Bishop, PhD; Andrea Murphy, PharmD; James Barker, PhD; Bobbi Morrison, PhD; Jason Perepelkin, PhD; Neil MacKinnon, PhD; Jeannette Sandiford, BSP; Stephanie Gimblett, BBA (Candidate)

OBJECTIVES: Quality-related events (e.g., medication errors, near misses) (QREs) can significantly impact pharmacies, pharmacists and patients. Yet, research exploring QREs within community pharmacies is limited. This research explores community pharmacist comfort with reporting QREs (i.e., informally with colleagues, formally at staff meetings) of different patient outcomes (i.e., near miss, no harm, short-term discomfort, long-term discomfort) and whether correct dispensing procedures were followed (i.e., followed, not aware, ignored).

METHODS: $\quad$ Questionnaires were mailed to 962 Saskatchewan pharmacists in the fall of 2013 as part the ComPASS (Community Pharmacists Advancing Safety in Saskatchewan) initiative. Responses were captured using 5-point scales and open-ended questions. Descriptive statistics, paired-sample tests and thematic analysis were performed in the preliminary analysis.

RESULTS: $\quad$ A total of 277 (28.8\%) questionnaires were returned. Pharmacists perceived high comfort levels with: 1) discussing QREs regardless of severity level if an error was made while following existing dispensing procedures; and 2) discussing near misses and no patient harm QREs when unaware of the specific dispensing procedures. Comfort level was low when discussing QREs, regardless of severity, if the current dispensing procedures were ignored. Pharmacists were significantly $(p \leq .01)$ more comfortable discussing QREs informally versus at staff meetings regardless of QRE severity or if correct dispensing procedures were followed.

CONCLUSIONS: Comfort level of QRE reporting can be moderated by the severity of the error, whether dispensing procedures were followed and the context in which QREs are discussed. The results support the continued need for a research agenda focusing on the complex factors that influence pharmacist reporting of QREs. 


\section{Preliminary findings of a study of experience with prescriptive authority (PA) services among Saskatchewan community pharmacists}

Rahul Jain, MSc (Candidate); Roy Dobson, BScPharm, MBA, PhD

ABSTRACT:

In recent years, a significant change in the pharmacist's scope of practice is the expansion of prescriptive authority (PA). In Saskatchewan, pharmacists adopted an interdependent prescribing model to support interprofessional collaboration, public safety though the optimal use of drug therapy and the optimization of pharmacy competencies. In acquiring this new prescriptive authority, the community pharmacist also assumes new responsibilities and obligations, as well as transforming their relationships with patients and physicians. The purpose of this research is to assess the impact of prescriptive authority (PA) services (Level 1 and Minor Ailments Prescribing) on the traditional professional practices and workload of community pharmacists. To gain a better understanding of how pharmacists are responding to new and evolving models of practice, this study proposes to measure their experiences with PA services and its effect on professional practice. To investigate the study's research questions, a cross-sectional study using a mail-in questionnaire with an online option was initiated. All registered community pharmacists of Saskatchewan (998) were asked to participate. Data collection is in progress this time. Preliminary results will be reported at the June 2014 CPhA Conference. 


\section{Preventing misunderstanding of medication labels: Establishment of a multilingual medication labeling service that can be provided by all staff, with the use of existing resources}

Yuen Ming Tan, BPharm, MSc (Project Management); Tuck Seng Wu, BScPhm, MHSM

OBJECTIVES: Estimate the need for translated medication labels in Chinese, Malay and Tamil languages for patients in this hospital. Find out the impact on patients' understanding with additional translated medication instructions. Establish a multilingual medication labeling service for patients receiving medications from all pharmacy sections of this hospital, once the need is established. Find out the usefulness of this service to patients post-implementation.

METHODS: $\quad$ Patients and staff were engaged to establish needs and preferences. Frequently used instructions for commonly dispensed dosage forms were translated. The impact of translated instructions on patient understanding was also evaluated using a simple test. Various label designs and workflows were trialed; until the best design and workflow were agreed upon. Patients who received this service were surveyed postimplementation.

RESULTS: $\quad$ A staggering $68 \%$ and $85 \%$ of patients aged 45 years and older ( $n=81$ ) who were interviewed reported being unable to read English and having difficulty in understanding their medication labels respectively. Results of a simple test showed that having translated instructions improved patient understanding from 33\% to $87 \%$ of patients interpreting instructions correctly. Engagement of patients and staff showed the need and desire for a multilingual labeling service. Innovative ideas were trialed and successful establishment of this service, that can be provided by all staff and understood by patients ( $99 \%$ ( $n=233$ ) of patients found this service useful), was established. Many positive patient comments received.

CONCLUSIONS: The need for standardized translated medication instructions was established in our hospital. A multilingual medication labeling service was successfully implemented; with good feedback from patients. 


\section{The clinical pharmacist as a team member within a Pulmonary Rehabilitation Program (PRP)}

Manjit Bains, BScPharm; Ashley Bond, BScPharm (Candidate); Louis Chute, BScPharm (Candidate); Jaclyn Deonarine, BScPharm (Candidate); Kristina Paunovic, BScPharm; Katie Peterson, BScPharm (Candidate); Kristine Petrasko, BScPharm, CRE; Megan Scott, BScPharm (Candidate)

OBJECTIVES: $\quad$ Pulmonary Rehabilitation (PR) uses an interprofessional approach to address clients with chronic obstructive pulmonary disease (COPD). The Regional PRP program located in Winnipeg, MB offers PR at three sites, with funding for one 0.4 full-time equivalent (FTE) pharmacist. The role of the pharmacist in the PRP is new and requires development to facilitate engagement and collaboration amongst the team. Initially, the pharmacist will focus the majority of time at one site and will support prioritized reviews and education to the remaining sites. As PRP is a time-limited outpatient program, the pharmacist's role must include strategy for seamless community transition. Our goal is to clarify the role of the pharmacist as a PRP team member. Once clarified, the value of the role will be measured through key performance indicators with the goal to expand to full clinical pharmacist support for the entire PRP.

METHODS: $\quad$ Using a PDSA approach, team involvement processes will be implemented, evaluated and refined. The scope of practice of the pharmacist within the PRP will be described by:

- Medication Management through interprofessional team collaboration

- Development of patient specific COPD Action Plans

- Tools developed for assessment and education

- Outreach strategies to create a seamless community transition

RESULTS: $\quad$ Pharmacists add significant value to the care of patients with COPD, contributing to overall better patient management. We propose that increasing the understanding of the pharmacist's role in a PRP will help support future funding requests.

CONCLUSIONS: There is currently a lack of pharmacist involvement in PRPs across Canada. We feel that pharmacist involvement on PRP teams is essential to optimize patient care. Further research on defined cost effectiveness is warranted. 


\section{The validity of information-sharing about pharmacists' education, skills and scope of practice in informing public perceptions}

Jason Perepelkin, PhD; Melissa Abramovic, BSP

OBJECTIVES: Recent public opinion polls have consistently ranked pharmacists as highly trusted health care professionals, but the reasoning for this ranking continues to remain vague and inconclusive to many. One common assumption is that the ranking is due in part to the limited expectations the public has of the profession. The purpose of this research, therefore, was to determine whether the public's opinion of pharmacists would change if they knew more about or could more deliberately assess the duties pharmacists were capable of performing. Specifically, this study attempted to determine whether public awareness for a pharmacist's counseling duties could have an impact on the publics perception and evaluation of a pharmacists performance and effectiveness in relation to those counseling activities.

METHODS: $\quad$ Participants were shown a video of a counseling session, then asked to assess the session via a self-administered questionnaire; then participants were shown a video describing pharmacists formal education and scope-of-practice. Then participants were shown the same counseling session video and asked to assess the counseling session.

RESULTS: $\quad$ A total of 381 individuals participated. Following the first two videos, respondents reported very good/excellent knowledge of pharmacists' education/training (63.6\%), tasks a pharmacist completed to fill a prescription (56.6\%), what should be included in a counseling session (67.0\%) and pharmacists scope-of-practice (61.0\%). Following the video on pharmacists' education and scope-of-practice, in which most (90.5\%) reported the video increased their knowledge of pharmacy and pharmacists, respondents reported a more favourable impression of the pharmacist.

CONCLUSIONS: Results show that PECs can enhance public perception of pharmacists, yet few (18.3\%) respondents reported being exposed to any PEC in the previous 12 months, despite numerous campaigns being conducted during this time. 


\section{Public attitudes of community pharmacy reward points and incentive programs}

Jason Perepelkin, PhD

OBJECTIVES:

Reward points and incentive (RPI) programs are used in many competitive environments. What is unique to community pharmacy is that patients are consuming health care products and services and many times the patient is not the person ultimately paying for the medication. Some argue that RPIs may negatively impact patient care. However, none of the research published has included insight from members of the general public; the data collected almost exclusively came from members of the profession. This study sought to understand the attitudes of the public in regard to RPIs.

METHODS: $\quad$ A survey of members of the public using telephone interviews was conducted in January 2014 in Saskatchewan. The questionnaire used was designed specifically for this study and contained 30 Likert-scale items in addition to demographic indicators.

RESULTS: $\quad$ A total of 401 responses were collected. The majority (91.5\%) of respondents reported having a regular community pharmacy, with half (49.6\%) having a RPI card. Two-thirds (64.8\%) disagreed that pharmacists that provide RPIs were less trustworthy than those that did not and few (15.4\%) reported that their choice of pharmacy was influenced by whether or not RPIs were provided for prescription medications. Furthermore, onequarter (26.4\%) felt they should receive RPIs for using a specific pharmacy, while less than half (37.9\%) felt it was ethical for pharmacists to provide RPIs for prescription purchases. As well, few (20.0\%) felt banning RPIs would improve the image/reputations of pharmacists or that banning RPIs is in the best interest of the public (22.7\%).

CONCLUSIONS: This study is the first known study to examine public attitudes of pharmacy reward points/incentives. Findings can be used in connection with previous studies on pharmacist perceptions to help inform policy decisions. 


\section{Understanding pharmacists' personality traits using the Big Five Inventory}

Meagen Rosenthal, MA, PhD (Candidate); Carlo Marra, PharmD, PhD; Bob Nakagawa, Registrar of the College of Pharmacists of British Columbia; Nicole Tsao, MScPharm, BScPharm (Candidate); Larry Lynd, PhD; Ross Tsuyuki, PharmD

OBJECTIVES: The psychology literature shows a relationship between personality traits and success in the work environment. To characterize the personality of pharmacists in British Columbia using the Big Five Inventory $(\mathrm{BFI})$ and relating this to adoption of advanced practice opportunities.

METHODS: $\quad$ An online survey was distributed to all pharmacists registered in British Columbia. The survey evaluated demographics, primary work setting and self-identified role, organizational culture profile and the BFI. This abstract will focus on results from the BFI. The analysis included the use of descriptive statistics (mean, median and mode) and inferential statistics examining mean differences between sub-groups and several advanced practice proxies such as prescription adaptation.

RESULTS: $\quad$ A total of 945 completed surveys were returned for a response rate of $19 \%$. The majority of respondents identified their primary work setting as a chain community pharmacy, self-identified, as staff pharmacists and $62 \%$ of respondents were female. The mean BFI scores (SD) were as follows (each out of 5 , with 5 indicating strong expression of the trait): extraversion 3.34 (0.68), agreeableness 4.09 (0.49), conscientiousness 4.23 (0.52), neuroticism 2.38 (0.67) and openness 3.45 (0.55). Sub-group differences were noted between practice setting and openness and practice role (e.g., staff pharmacist) and extraversion, neuroticism and openness. Differences between BFI scores and the number of prescription adaptations, immunizations provided, medication reviews conducted per month were observed.

CONCLUSIONS: The results suggest possibly important differences between personality traits and pharmacists' role, as well as differences in personality traits and the number of advanced practice services provided to patients. 


\section{Understanding pharmacy culture using the Organizational Culture Profile}

Meagen Rosenthal, MA, PhD (Candidate); Carlo Marra, PharmD, PhD; Bob Nakagawa, Registrar of the College of Pharmacists of British Columbia; Nicole Tsao, MScPharm, BScPharm (Candidate); Ross Tsuyuki, PharmD; Larry Lynd, PhD

OBJECTIVES: The business and knowledge translation literature state that understanding the culture of an organization is key to implementing change. To characterize the culture of pharmacists in relation to adopting advanced practice opportunities using the organizational culture profile (OCP).

METHODS: $\quad$ An online survey was distributed to all pharmacists registered in British Columbia. The survey was composed of three sections:

1) pharmacist demographics, primary work setting and self-identified role,

2) OCP,

3) Big Five Inventory.

This abstract will focus on results from sections one and two. Analyses included the use of descriptive statistics (mean, median and mode) and inferential statistics examining mean differences between the OCP and sub-groups and several advanced practice measures.

RESULTS: $\quad$ A total of 945 completed surveys were returned for a response rate of 19\%. The majority of respondents identified their primary work setting as a chain pharmacy, they self-identified as staff pharmacists and $62 \%$ of respondents were female. The mean OCP scores (SD) of respondents were as follows (each $/ 5$ with 5 indicating strong agreement): innovation 3.14 (0.80), supportiveness 3.48 (0.92), social responsibility 3.37 (0.81), competitiveness 3.44 (0.82), stability 3.28 (0.60), performance orientation 3.52 (0.76) and reward orientation 3.04 (0.90). Sub-group differences were noted between pharmacist practice setting and pharmacist role (e,g., staff pharmacist) and OCP scores. Differences were noted between OCP scores and the number of immunizations provided and medication reviews conducted per month.

CONCLUSIONS: These results suggest that organizational culture may explain adoption of advanced practice opportunities by pharmacists. 


\section{Pharmacists' working conditions in British Columbia}

Nicole Tsao, MScPharm, BScPharm (Candidate); Carlo Marra, PharmD, PhD; Kathy Li, PhD; Larry Lynd, PhD; Bob Nakagawa, Registrar of the College of Pharmacists of British Columbia

OBJECTIVES: Over the last decade, in addition to increasing volumes of prescriptions, pharmacists are undertaking an expanded clinical role, providing medication management services, prescription adaptations and immunizations. There has not been a study in British Columbia $(B C)$ to determine whether current working conditions effectively support safe patient care practices. To identify key concerns of BC pharmacists about workplace conditions, identify and quantify workplace setting and practices that impact patient safety.

METHODS: $\quad$ An online survey was developed, based on a survey done in Oregon, USA and distributed to all BC College of Pharmacists registrants by email. Responses were collected from October 1st - November 10th, 2013. The survey consisted of demographics questions, questions about respondents' pharmacy practice site and open-ended questions.

RESULTS:

$1241 / 5300$ (23\%) of pharmacists responded, $78 \%$ worked in the community pharmacy setting (58\% chain, 19\% independent). 69\% were staff pharmacists and $28 \%$ were pharmacy managers. Overall, 485/1017 (48\%) of respondents reported not having enough time at work for breaks or lunch and 457/1017 (45\%) reported not being satisfied with the amount of time they have to do their jobs. When asked whether the respondents' practice site had adequate pharmacist and technician staff to provide safe and effective patient care, the majority agreed there were adequate pharmacists but not enough technicians. Responses to open-ended questions revealed that pharmacists are faced with increased pressures to provide more clinical services without adequate support.

CONCLUSIONS: Pharmacists feel that expanded scope of practice without additional pharmacy support staff can adversely impact patient care. 


\section{Systematic review of pharmacist role and interventions in long-term care facilities}

Cheryl Sadowski, BScPharm, PharmD, FCSHP; Sujin Kim, BScPharm (Candidate); Scot Simpson, BSP, PharmD; Patricia Chatterley, MLIS

OBJECTIVES: The purpose is to review the effect of pharmacist-based interventions on outcomes of older adults living in long-term care facilities (LTCF).

METHODS: $\quad$ We searched MEDLINE, Embase, International Pharmaceutical Abstracts, the Cochrane Library all on the Ovid interface and EBSCO CINAHL, from inception to June 2013. Two authors reviewed all articles and abstracted eligible articles independently using a standard form. Articles were included if they met the criteria:

1) interventions were pharmacist-led or the pharmacist was part of a team,

2) controlled (randomized or non-randomized) or quasi-experimental studies and

3) setting of LTCF.

Discrepancies were resolved by consensus. Outcomes were categorized into clinical, humanistic, or economic and information summarized semi-quantitatively.

RESULTS: $\quad$ A total of 568 articles were identified in the search; 29 studies met inclusion criteria. All studies used pharmacist-led medication reviews as the sole activity or part of a multi-component intervention. Medication review had a statistically significant effect on medication use in 11 trials as demonstrated by $3-10 \%$ reductions in the number of medication changes. High-risk medication use (e.g. benzodiazepines) was reduced in 5 studies by up to $9.6 \%$. Five studies showed statistically significant improvement on hospitalizations ( $R R R=0.84$ ) and drug cost savings (up to $4.4 \%$ ).

CONCLUSIONS: Pharmacists are engaged in a diversity of resident care activities in LTCF. Pharmacist involvement not only enhances overall usage of medications and clinical outcomes but may also reduce medical expenditures. 


\section{Inclusion of information regarding older adults in drug product monographs}

Cheryl Sadowski, BScPharm, PharmD, FCSHP; Ken Cor, BSc Eng, PhD

OBJECTIVES: $\quad$ The purpose of this study was to examine newly marketed or approved drug product monographs provided by Health Canada (starting from 1994) to determine the extent and type of data included about geriatrics.

METHODS: $\quad$ Product monographs were identified by assistance of the Notice of Compliance database and Drug Product Directorate (Health Canada). Monographs indicating that older adults could use the medication or biological product were included in the review. Information related to the geriatric population in the following categories of the monographs was abstracted: general information, pharmacokinetics, pharmacodynamics, precautions, warnings, adverse effects/events, dosage information, clinical trial inclusion and geriatric specific clinical trials. Data were analyzed descriptively.

RESULTS: $\quad$ A total of 296 drug monographs were evaluated from 122 different AHFS drug categories and 75 different drug manufacturing companies. Two-hundred-eightytwo (95\%) included general information on the geriatric population, mostly under Precautions ( $n=251,85 \%)$. Two hundred (67.6\%) and 6 (2\%) included information on geriatric pharmacokinetics and pharmacodynamics, respectively. For safety concerns, $31(10 \%)$ of the product monographs contained information on adverse effects in the geriatric population.

CONCLUSIONS: Many drug product monographs have limited information that is specific to the elderly population. Most monographs include precaution information that is quite generic, e.g., information about lack of research or minimizing use and dose in older adults. 


\section{Measuring the value of community pharmacists' provision of professional services: A methodology using pharmacy billing data}

Nancy Winslade, BScPhm, PharmD, MHPE; Robyn Tamblyn, PhD; Danielle Fagnan, MSc; Sherry Shi, MSc; Teresa Moraga, MSc

OBJECTIVES: As pharmacists are increasingly authorized and funded to provide professional services, we investigated whether community pharmacist's billing data could be used to determine the appropriateness and impact of the provision of selected professional services.

METHODS: $\quad$ Previous work has used Quebec's community pharmacy billing data to retrospectively measure pharmacy-level performance on targeted quality-of-care indicators. As part of a trial evaluating performance feedback, these methodologies were used to calculate real-time pharmacy-level rates of dispensings to patients with over or under-use of asthma or hypertension medications respectively. Subsequent analysis determined pharmacy-level rates of provision of targeted pharmaceutical opinions as one measure of appropriate provision of these professional services.

RESULTS: Calculation of pharmacy-level performance on the specific indicators required modification of previous methodologies to ensure consistency with provincial reimbursement policies. The majority of consenting pharmacies had the information required to allow performance calculation (hypertension 1814 of 1815 pharmacies, asthma 1598 of 1814 pharmacies). Changes in pharmacy-level performance on the indicators were measured over the study period as well as significant increases in pharmacy-level billing rates for asthma-specific pharmaceutical opinions in response to performance feedback.

CONCLUSIONS: The methodology met key criteria including allowing calculation of performance for the majority of pharmacies, detecting changes in both indicator performance and provision-rates of targeted professional services and measuring the impact of these professional services on indicator performance. Billings are currently being linked at the anonymized patient-specific level to determine if appropriate patients received the services. Elements required in pharmacist's-services billing data are being identified for expansion of the methodology-use beyond Quebec. 


\section{Pharmacists' perceptions of the impact of Computerized Prescriber Order Entry (CPOE) on their practice}

Heather Kertland, PharmD, FCSHP; Elaine Tom, BScPhm

OBJECTIVES:

CPOE is advocated as a patient safety initiative. The impact of this technology has been measured by metrics such as reduced medication turnaround time and decreased inappropriate antibiotic choices. We wished to determine how this technology impacted a pharmacists practice.

METHODS: $\quad$ Pharmacists who worked a minimum of six months prior to and following the implementation of CPOE were invited to participate in one-on-one interviews that were conducted using a semi-structured guide by a trained interviewer. Two investigators analyzed the verbatim transcripts to identify themes. The analysis was validated with a group of eligible pharmacists.

RESULTS: $\quad$ Pharmacists perceived that CPOE had the greatest impact on their role in the medication system, direct patient care and interdisciplinary team collaboration. For the first two roles, major themes were improved efficiency (e.g., prioritization of orders, timely medication order review, quicker resolution of problem orders, complete medication profile was available for patient assessment) and safety (easier to determine appropriate timing of next dose, no illegible writing, increased time to resolve complex problems). Interdisciplinary team communication, particularly order clarification with physicians, was facilitated by the timeliness of order review. The use of technology revealed new safety concerns that emphasized the essential role that a pharmacist has to ensure patient safety.

CONCLUSIONS: Overall, pharmacists perceived that the introduction of CPOE had a positive impact on their practice. It permitted a more efficient assessment of medication and patient information, providing additional time to identify and resolve drug therapy problems. New types of safety concerns were detected which needed to be addressed. 